\title{
Disentangling timing and amplitude errors in streamflow simulations
}

\author{
Simon Paul Seibert, Uwe Ehret, and Erwin Zehe \\ Chair of Hydrology, Institute for Water and River Basin Management, Karlsruhe Institute of Technology (KIT), \\ Kaiserstrasse 12, 76131 Karlsruhe, Germany \\ Correspondence to: Uwe Ehret (uwe.ehret@kit.edu)
}

Received: 24 March 2016 - Published in Hydrol. Earth Syst. Sci. Discuss.: 30 March 2016

Revised: 13 July 2016 - Accepted: 16 July 2016 - Published: 12 September 2016

\begin{abstract}
This article introduces an improvement in the Series Distance (SD) approach for the improved discrimination and visualization of timing and magnitude uncertainties in streamflow simulations. SD emulates visual hydrograph comparison by distinguishing periods of low flow and periods of rise and recession in hydrological events. Within these periods, it determines the distance of two hydrographs not between points of equal time but between points that are hydrologically similar. The improvement comprises an automated procedure to emulate visual pattern matching, i.e. the determination of an optimal level of generalization when comparing two hydrographs, a scaled error model which is better applicable across large discharge ranges than its nonscaled counterpart, and "error dressing", a concept to construct uncertainty ranges around deterministic simulations or forecasts. Error dressing includes an approach to sample empirical error distributions by increasing variance contribution, which can be extended from standard one-dimensional distributions to the two-dimensional distributions of combined time and magnitude errors provided by SD.

In a case study we apply both the SD concept and a benchmark model (BM) based on standard magnitude errors to a 6-year time series of observations and simulations from a small alpine catchment. Time-magnitude error characteristics for low flow and rising and falling limbs of events were substantially different. Their separate treatment within SD therefore preserves useful information which can be used for differentiated model diagnostics, and which is not contained in standard criteria like the Nash-Sutcliffe efficiency. Construction of uncertainty ranges based on the magnitude of errors of the BM approach and the combined time and magnitude errors of the SD approach revealed that the BM-derived
\end{abstract}

ranges were visually narrower and statistically superior to the SD ranges. This suggests that the combined use of time and magnitude errors to construct uncertainty envelopes implies a trade-off between the added value of explicitly considering timing errors and the associated, inevitable time-spreading effect which inflates the related uncertainty ranges. Which effect dominates depends on the characteristics of timing errors in the hydrographs at hand. Our findings confirm that Series Distance is an elaborated concept for the comparison of simulated and observed streamflow time series which can be used for detailed hydrological analysis and model diagnostics and to inform us about uncertainties related to hydrological predictions.

\section{Introduction}

Manifold epistemic and aleatory uncertainties make the simulation of streamflow a fairly uncertain task. The assessment of uncertainties, i.e. quantification, evaluation, and communication, is thus of great concern in decision making, model evaluation, the design of technical structures like flood protection dams or weirs, and many other issues. The quantification and evaluation of uncertainties typically involves the comparison of simulated and observed rainfall-runoff data.

For this purpose, visual hydrograph inspection is still the most widely used technique in hydrology as it allows for the simultaneous consideration of various aspects such as the occurrence of hydrological rainfall-runoff events, the timing of peaks and troughs, the agreement in shape, and the comparison of individual rising or falling limbs within an event. The main strength of visual hydrograph comparison results 
from the human ability to identify and compare matching, i.e. hydrologically similar parts of hydrographs ("to compare apples with apples") and particularly to discriminate vertical (magnitude) and horizontal (timing) agreement of hydrographs. Whereas the former implies that rising and falling limbs of the two time series are intuitively and meaningfully matched before they are compared, the latter refers to a joint but yet individual consideration of timing and magnitude errors. Visual hydrograph inspection is hence a powerful yet demanding evaluation technique which is still rather difficult to mimic by automated methods. Clear disadvantages of visual hydrograph inspection, however, are its subjectivity and that its application is restricted to a limited number of events.

\subsection{Single and multiple criteria for hydrograph evaluation}

To overcome this shortcoming, a large number of numerical criteria (Nash and Sutcliffe, 1970; Legates and McCabe, 1999; Pachepsky et al., 2006; Dawson et al., 2007; Laio and Tamea, 2007; Bennett et al., 2013) have been proposed. However, each criterion typically evaluates only one or just a few hydrograph aspects and there is no "one size fits all" solution available. For this reason different attempts have been undertaken to compare expert judgement and automated criteria (Crochemore et al., 2014) and to establish model evaluation guidelines (e.g. Moriasi et al., 2007; Biondi et al., 2012; Harmel et al., 2014). Key points of related guidelines typically include the statement that the choice of the metric should depend (i) on the modelling purpose, (ii) on the modelling mode (calibration, validation, simulation, or forecast), and (iii) on the model resolution (time stepping, spatial resolution). Further, most authors recommend the combination of several, preferably orthogonal criteria, which might imply combined application of absolute and relative criteria (Willmott, 1981). Hence, within the last decade several multi-criteria approaches for model calibration and evaluation have been proposed (Gupta et al., 1998; Boyle et al., 2000; Vrugt et al., 2003; Efstratiadis and Koutsoyiannis, 2010; Kollat et al., 2012), which combine different performance criteria and/or evaluation against hydrological signatures such as the shape of the flow duration curve (Euser et al., 2013; Hrachowitz et al., 2014). Even approaches aiming to mimic visual hydrograph comparison were developed. These include multicomponent mapping (Pappenberger and Beven, 2004), self-organizing maps (Reusser et al., 2009), wavelets (Liu et al., 2011), the hydrograph matching algorithm (Ewen, 2011), and the "Peak-Box" approach for the interpretation and verification of operational ensemble peakflow forecasts (Zappa et al., 2013). Despite this considerable progress, many practical and scientific applications (Haag et al., 2005; Gassmann et al., 2013; Seibert et al., 2014; Wrede et al., 2014; Kelleher et al., 2015; Zhang et al., 2016) still rely on simple mean squared error (MSE) type distance metrics such as the long-established Nash-Sutcliffe ef- ficiency (NASH) or the root mean squared error (RMSE) even though their shortcomings are well known (Seibert, 2001; Schaefli and Gupta, 2007; Gupta et al., 2009).

A less recognized issue of MSE-type criteria is that these compare points with identical abscissa, i.e. at the same position in time. This means that points in the observation are "vertically" compared to points in the simulation (in the following we refer to them as vertical metrics). The problem with this is that small errors in timing may be expressed as large errors in magnitude. It is obvious that neither individual criteria nor the combination of different vertical metrics within a multi-objective approach can compensate for this.

\subsection{Uncertainty assessment and model diagnostics - learning from model deficiencies}

Just as with performance criteria, many methods related to the quantification, visualization, and communication of uncertainties were developed in recent decades, and the value of knowledge about simulation uncertainty is now generally acknowledged. The range of methods is large and comprises manifold probabilistic and non-probabilistic approaches. Probabilistic concepts, for instance, include the total model uncertainty concept (Montanari and Grossi, 2008), methods based on Bayes' theorem (Krzysztofowicz, 1999; Krzysztofowicz and Kelly, 2000), and various ensemble techniques (Roulston and Smith, 2003; Georgakakos et al., 2004; Cloke and Pappenberger, 2008). Non-probabilistic methods include the generalized likelihood uncertainty estimation (GLUE) (Beven and Binley, 1992), possibilistic methods (Jacquin and Shamseldin, 2007), or approaches applying fuzzy-set theory (Nasseri et al., 2014). Uncertainty assessment is a field of ongoing research, and so far there is no generally accepted technique available. The most important points of criticism of the non-probabilistic methods are their subjectivity and their inconsistency with probabilistic approaches when these are applied to cases which can be explicitly answered using statistical approaches (Stedinger et al., 2008). On the other hand, probabilistic approaches always rely on the assumptions of ergodicity and stationarity, which are rarely fulfilled in reality. A spin-off of uncertainty assessment is the field of model diagnostics, which ultimately aims to learn more about and from model deficiencies. Related approaches either analyse the temporal patterns of parameter identifiability (Wagener et al., 2003) or the coincidence of typical errors (Reusser et al., 2009) and parameter sensitivity (Reusser and Zehe, 2011) in streamflow simulation.

Motivated by the limitations of vertical distance metrics, Ehret and Zehe (2011) developed the Series Distance (SD) approach. SD is not a single equation but rather a concept designed for joint but separated assessment of timing and magnitude errors in streamflow simulations, either for events in distinct periods or the entire time series. "Joint but separated" means that both the time and magnitude distances 
between the observed and simulated hydrographs are determined for matching pairs of points in the event, but the two distances are kept separate. Such separate treatment is for instance desirable in flood forecasting, where errors in magnitude are relevant for dike defence, whereas errors in timing are crucial for reservoir operation. The separation of timing and magnitude errors is further helpful for improving model diagnostics as they point towards different deficiencies in the model structure.

Here we present substantial improvements (Sect. 2) to the original approach of Ehret and Zehe (2011), particularly the coarse-graining procedure. We furthermore introduce a heuristic approach to visualize timing and magnitude uncertainties in streamflow simulations by constructing two-dimensional uncertainty ranges in Sect. 3. Related to that, we provide and test several quality criteria to evaluate deterministic uncertainty ranges. The skill of uncertainty ranges is still rarely evaluated in hydrology (Franz and Hogue, 2011), and most of the available methods such as rank probability scores (Duan et al., 2007), rank histograms, or the usage of different moments of the probability density function (De Lannoy et al., 2006) were developed in climatology (Gneiting et al., 2008; Franz and Hogue, 2011). These approaches typically quantify ensemble spread and thus are probabilistic approaches to evaluate uncertainty estimation. To our knowledge only few deterministic approaches, e.g. categorical statistics such as the Brier score or contingency tables or combinations of deterministic and probabilistic approaches (Shrestha et al., 2009), are available. In Sect. 4 we test the feasibility of the advanced SD approach in a case study and compare it to a standard benchmark error model. Section 5 contains the results and discussion, Sect. 6 the related conclusions. To foster the use of the SD approach, we publish the SD (Matlab) code, licensed under Creative Commons license BY-NCSA 4.0, together with a ready-to-use sample data set alongside this manuscript. It is accessible via a GitHub repository https://github.com/KIT-HYD/SeriesDistance (Ehret and Seibert, 2016).

\section{Series distance - concept and modifications}

SD was developed to resemble the strengths of visual hydrograph inspection in an automated procedure, which typically rests on the following premises (Ehret and Zehe, 2011):

- Hydrographs contain individual events separated by periods of low flow.

- Events are composed of rising and falling limbs or segments which are separated by peaks and troughs.

- These different parts of event hydrographs reflect different hydrometeorological processes and should be compared individually, so as to not compare apples with oranges. This is of particular importance if the simulated

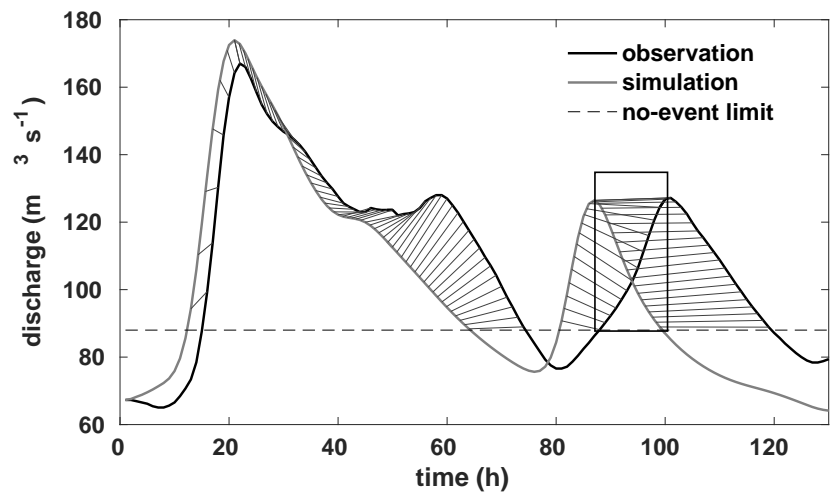

Figure 1. Time series of observed (black) and simulated (grey) discharge during a hydrological event. The horizontal line represents a user-specific threshold which differentiates between event and nonevent periods. The light grey lines represent the Series Distance connectors linking hydrologically comparable points in the two time series. Time and magnitude distances are calculated between these points. The black rectangle highlights time steps where a part of the recession of the simulation overlaps with a rising part of the observation (figure from Ehret and Zehe, 2011).

(sim in the following) and observed (obs in the following) hydrographs do belong to different parts of the hydrograph at the same time step $t$ (compare black rectangle in Fig. 1).

- A comprehensive evaluation of the agreement of matching rising and falling limbs of two hydrographs requires consideration of both errors in timing and magnitude as this better informs us about ways to improve the model. A simulated rising limb can, for example, match perfectly with its observed counterpart with respect to values but occur systematically too early or too late, which would indicate the need to adjust model parameters related to runoff concentration and flood routing or to improve the related model components.

- A comprehensive comparison of sim and obs should also provide information on the overall agreement with respect to the occurrence of relevant events and times of low flow. This is typically expressed by contingency tables, which contain information about correctly predicted, missed, and falsely predicted events.

These criteria listed above inform about different error sources, and their individual evaluation therefore provides useful information for a targeted model improvement. As SD accounts for all of these aspects, it is not a single formula but rather a procedure which includes the following steps. For each step, the main innovations are described in detail in the sections below.

- Hydrograph preprocessing (Sect. 2.1). New: routines to create gap-free, non-negative time series and to filter irrelevant fluctuations. 
- Identification and pairing of events (Sect. 2.2). New: routines to read user-specified events and to treat the entire time series as a single, long event.

- Identification, matching, and coarse-graining of segments (Sect. 2.3): New: this part has been completely reworked and now applies the coarse-graining procedure.

- Calculation of the distance between matching segments with respect to both timing and magnitude (Sect. 2.4). This is the core of SD, and it is important to note that the distances are computed between points of the hydrographs considered to be hydrologically similar. New: routines to calculate a scaled magnitude error.

- Calculation of a contingency table which counts matching, missing, and false events. No changes.

\subsection{Hydrograph preprocessing}

The application of SD usually requires some preprocessing to assure gap-free and non-negative time series of equal length; related routines are now included in the SD code. Further routines are available for the adjustment of consecutive identical values; the identification of rising and falling limbs requires non-zero gradients and for time series smoothing, which is often necessary due to the presence of sensor-related non-relevant microsegments. Smoothing is based on the Douglas-Peucker algorithm (Douglas and Peucker, 1973), which preserves extremes but filters the noise (Ehret, 2016). Preprocessing also involves the identification of segments, i.e. contiguous periods of rise or fall in the hydrograph. This is based on the slope of the hydrograph computed between two successive time steps.

\subsection{Identification and pairing of events}

For many aspects of hydrology such as flood forecasting or studies of rainfall-runoff transformation, it is useful to consider a hydrograph as a succession of distinct events, usually triggered by rainfall events, separated by periods of low flow. As SD is based on the concept of comparing similar parts of obs and sim hydrographs, it ideally also involves the steps of identifying events both in the obs and sim time series and then relating the resulting events between the series. On this level, the general agreement of the two series is evaluated with a contingency table, which counts the number of hits (observed events that have a matching simulated counterpart), misses (observed events without a simulated counterpart), and false alarms (simulated events without an observed counterpart). This is also the basis for the further steps of the SD procedure: only for matching pairs of obssim events can matching segments of rise and fall within the events be identified and the combined time-magnitude error be computed. For misses, false alarms, and periods of low flow this is not possible. For these cases, the best indicator of hydrological similarity in obs and sim is similarity in time; i.e. the distance between the observed and simulated hydrograph can be computed with a standard vertical distance measure. The detection of events in hydrographs and their subsequent pairing, however, is not trivial and has to our knowledge not yet been solved in an automated and generalized way. The original version of SD applied a simple no-event threshold (see Fig. 1) which, however, often produced unsatisfactory results in the form of many non-intuitive misses or false alarms if the events peaked just above or below the threshold. To overcome these limitations, two further options are now included in SD. The first allows the reading of event start and end points and matching obs and sim events from user-provided lists. This "event mode" option allows users to apply any desired event detection method, such as those proposed by Blume et al. (2007), Seibert et al. (2016), or Merz and Blöschl (2009), and is recommended if a clear distinction between events and low flow is important. If the identification of events is either not possible or relevant, both the obs and sim time series can be treated as two single, long, matching events, and the steps of segment identification and matching as described in the next section are applied to the entire time series. Despite its simplicity, this "continuous mode" has been shown to work well in the authors' opinion after applying the SD approach to different discharge time series in both the event and the continuous mode. Shown to work well in this context means even in the continuous mode, SD linked parts of obs and sim time series that visually appeared to be matching segments within matching events. Since this is difficult to show in a simple graph or statistic, we provide the SD code and test data together with the article.

\subsection{Pattern matching: identification, matching, and coarse-graining of segments}

This section describes the core of the SD concept, i.e. the way to identify, within a matching pair of an observed and a simulated event, hydrologically comparable points of the hydrographs in order to quantify their distance in magnitude and time. This pattern matching procedure has been substantially improved in the new version of SD and is therefore described in detail here.

The term "hydrologically comparable" relates to how a hydrologist would visually compare hydrographs and includes several aspects and constraints. The first constraint is based on the perception that even if hydrological simulations may deviate from the observations in magnitude or timing, their temporal order is usually correct. Therefore, in SD, matching points are compared chronologically by preserving their temporal occurrence: the first point in obs is compared to the first in sim, the second to the second, the last to the last. Please note that this does not require the two events to be of equal length, as in $\mathrm{SD}$, the hydrograph is considered a polygon from which the points to compare can be sampled 
by linear interpolation without restriction to its edge nodes. This is explained in detail below. The second constraint relates to the slope of the hydrograph: to ensure hydrological consistency, points within rising segments of sim are only compared to points in rising segments of obs, and the same applies to falling segments. This creates a problem related to the within-event variability of the two hydrographs: it is easy to imagine a case in which the number of segments in the obs and sim event differs. This can be either due to sensor-related high-frequency micro fluctuations of the observations, which can create sequences of many short rising and falling segments, or to general deviations of the simulation from the observation, such as a double-peaked simulated event while the observed event is single-peaked. In visual hydrograph evaluation, a hydrologist will detect the dominant patterns of rise and fall in the two time series and identify matching segments by doing two things: filtering out short, non-relevant fluctuations and then relating the remaining ones by jointly evaluating their similarity in timing, duration, and slope. The stronger the overall disagreement of the obs and sim event, the more visual coarse-graining will be done before the hydrographs are finally compared, while at the same time the degree of coarse-graining will also influence the hydrologist's evaluation of the hydrograph agreement: the higher the required degree of coarse-graining, the smaller the agreement. In SD, these steps are emulated by iteratively maximizing an objective function: while increasingly coarse-graining the two events, their overall time and magnitude distance is evaluated. The final evaluation of agreement is then done on the level at which the optimal trade-off between coarse-graining and hydrograph distance occurs, i.e. where the objective function is minimal. The procedure consists of four steps and is explained in the following sections: (1) determination of segment properties, (2) equalizing the number of segments in the obs and sim event, (3) iterative coarse-graining, and (4) distance computation for the optimal coarse-graining level.

1. For each segment $i$ in the initial sequence of rises and falls of an event, its properties relevant for coarsegraining are determined: start and end time, duration $(\mathrm{d} t(i))$, and absolute magnitude change $(\mathrm{d} Q(i))$. From this the relative duration $\left(\mathrm{d} t^{*}(i)\right)$ and the relative magnitude change $\left(\mathrm{d} Q^{*}(i)\right)$ of each segment is calculated, i.e. its duration normalized by the total duration and its magnitude change normalized by the total sum of absolute magnitude changes of the entire event. $\mathrm{d} t^{*}(i)$ and $\mathrm{d} Q^{*}(i)$ are then used to determine the relative importance of each segment $\left(I_{\mathrm{SEG}}(i)\right)$ using the Euclidean distance (Eq. (1)). Taken together, all $I_{\mathrm{SEG}}(i)$ of the time series sum up to 1, and segments that are relevant, i.e. that are either very long and/or include large discharge changes, receive large values of $I_{\mathrm{SEG}}$.

$$
I_{\mathrm{SEG}}(i)=\sqrt{\mathrm{d} t^{* 2}(i)+\mathrm{d} Q^{* 2}(i)}
$$

2. If the number of segments in the obs and sim event differs, they are logically equalized by removing the required number from the event with the surplus. This is done with a directed, iterative aggregation of segments: the least relevant segment (the one with the smallest value of $I_{\mathrm{SEG}}$ ) is selected and assimilated by its two neighbouring segments. For instance, a small relevant rising segment will then be combined with its preceding and succeeding falling segment to a single, long, falling segment. For the new segment the properties are then determined; its relative importance is the sum of the previous three segments.

It is important to note that this procedure is a purely logical assimilation: the timing and magnitude of the points in the dissolved segment remain unchanged; they are only reassigned to the new and larger segment. This also implies that the meaning of coarse-graining in the context of SD is slightly different from its meanings in statistics and thermodynamics or in upscaling (Attinger, 2003; Neuweiler and King, 2002). In the first case, coarse-graining is synonymous with the aggregation and averaging of physical quantities; in the second, it is related to the preservation of heterogeneity effects upon aggregation. In the case of SD, it means that logical ordering properties are aggregated, while the absolute values of the timing and magnitude of the data are not changed.

Obviously, this procedure includes a false classification: the rising segment in the previous example is now hidden within a larger falling segment. This can be considered as the price of coarse-graining and can be quantified by the number of falsely classified edge nodes $\left(n_{\text {mod }}^{*}\right)$ of the time series. Therefore, $n_{\text {mod }}^{*}$ is a useful quantity to punish excessive coarse-graining in the objective function, Eq. (2).

3. With the number of segments in the obs and sim events equalized, their SD timing and magnitude distance can be computed. To this end, the first obs segment is compared to the first sim segment, the second to the second, etc. Since the segments can differ in length we here assume that for each segment pair, the appropriate number of points is evenly distributed along the segment duration and can thus be found by linear interpolation between the time series edge nodes. The first point in the obs segment is then connected to the first point in the sim segment, the second to the second, etc. For each connector its horizontal and vertical projection, i.e. length in time and magnitude, respectively, is determined (compare again Fig. 1), yielding the joint time and magnitude error of the particular point pair.

In the initial version of SD, the number of points for each segment pair was found by calculating the mean of the two relative durations, $I_{\mathrm{d} t}^{*}$, such that long-segment 
pairs received many points and the overall number of connector points of the time series equalled its number of edge nodes. In order to better emulate a hydrologist's perception of segment importance, in the current version of SD the number of points is determined by the mean relative importance $I_{\text {SEG }}$ (Eq. 1) of a segment pair. This assigns more points to (and hence puts more emphasis on) short but steeply rising segments while still preserving the same overall number of points.

At this point the result of the SD procedure - a twodimensional distribution of time and magnitude errors, separately for the rising and the falling segments - is available. However, in practice the problem of nonintuitive segment matching often spoils the results. Due to the constraint of time-ordered segment matching, any minor change in monotony within a rising or a falling limb that is only present in either the obs or sim event will produce a false matching of segments. The left panel in Fig. 2 illustrates this problem, where the first falling segment in the observed series (labelled with " 2 " in a square) corrupts segment matching: in chronological terms the steep flood rise in obs (" 3 " in a square) would be compared to the second rising segment in sim (" 3 " in a circle), which is obviously wrong. In this case, the SD time and magnitude distances will be very large, while visual comparison would most likely be done as shown in the right panel of Fig. 2 and yield good agreement.

We overcome this problem using iterative coarsegraining again: within the events, successively more segments are logically aggregated with their neighbours until finally the entire event consists of only two segments: one rise and one fall. Compared to the last step, in which we apply coarse-graining to either sim or obs in order to equalize the number of segments in the simulated and observed event, we here apply it simultaneously to the obs and sim event. Hence, an equal number of segments and unique segment matching is ensured. The final comparison of the two events is done for the coarse-graining step in which the total SD errors and the degree of coarse-graining together are small. Both requirements are considered in the coarse-graining objective function $(\theta)$. The latter consists of four criteria. The first two are as follows: (i) the number of edge nodes in falsely classified segments $\left(n_{\text {mod }}^{*}\right)$ and (ii) the cumulated importance of the dissolved segments ( $I_{\mathrm{SEG}, \mathrm{cum}}^{*}$ ). As discussed above, the false classifications inevitably occur during the aggregation of segments. Both criteria monotonically increase with the number of dissolved segments and therefore punish excessive coarsegraining. Further criteria are (iii) the $\mathrm{SD}$ timing $\left(E_{\mathrm{SD}, t}^{*}\right)$ and (iv) magnitude errors $\left(E_{\mathrm{SD}, Q}^{*}\right)$ summed up over all segments of the event. They are small when segments that are hydrologically similar, i.e. close in time, dura- tion, and magnitude, are compared. As in Eq. (1), each criterion is first normalized to the range of [0 1] and then combined using the Euclidean distance (Eq. (2)):

$\theta=\sqrt{\gamma_{1} n_{\mathrm{mod}}^{* 2}+\gamma_{2} I_{\mathrm{SEG}, \mathrm{cum}}^{* 2}+\gamma_{3} E_{\mathrm{SD}, t}^{* 2}+\gamma_{4} E_{\mathrm{SD}, Q}^{* 2}}$.

Note that $\theta$ also includes weighting factors $\left(\gamma_{1} \ldots \gamma_{4}\right)$ for each criterion, which allows for a user- or timeseries-specific adjustment of the objective function. Their setting is hence case-specific, with the constraint that $\gamma_{1} \ldots \gamma_{4}$ have to sum up to unity. For example, if the temporal agreement of segments is important, the weight for $E_{\mathrm{SD}, t}^{*}$ should be large. Setting $\gamma_{3}=1$ and all other weights to 0 will hence result in a vertical comparison of the time series, provided that the positions of the edge nodes are identical. The opposite case $\left(\gamma_{4}=1\right.$ and $\gamma_{1}=\gamma_{2}=\gamma_{3}=0$ ) minimizes vertical deviations which leads to horizontally extended SD connectors. Large weights for either $\gamma_{1}$ or $\gamma_{2}$ will prevent any logical aggregation and the pattern matching procedure will suggest the initial conditions as the best solution. Consequently, "extreme" parametrizations of $\theta$ are not meaningful as they will prevent the purpose of SD, which is to compare points which are hydrologically similar.

As can be seen in Fig. 2, dissolving a single segment can drastically change the events' overall SD time and magnitude distance. Also, as during the successive removal of segments in coarse-graining, it is impossible to predict which combination of segments dissolved in obs and sim will yield the best value of $\theta$; thus, all possible combinations are tested and the best is kept. If, e.g., both the obs and sim event consist of 10 segments, $10 \times 10$ combinations of segment dissolutions are tested (obs 1 with $\operatorname{sim}_{1}$, obs $_{1}$ with $\operatorname{sim}_{2}$, etc.). The coarse-graining scheme is thus computationally demanding. The combination with the minimum $\theta$ is kept and serves as the basis for the next segment reduction step in the coarsegraining procedure.

4. Once the coarse-graining is done, the optimal value of $\theta$ is available for each reduction step, starting with the initial number of segments and ending with two. In Fig. 3, this is shown for a three-peak event with initially 15 segments. As can be seen in the lower right panel, the value of the objective function is initially high: here segment matching is poor and as a result SD timing errors and thus $\theta$ are high (upper left panel). After dissolving three segments, agreement is much better (lower left panel) and $\theta$ is at its minimum. Further segment aggregation does not further decrease SD errors, but now the number of falsely classified nodes increases and leads to an increase in $\theta$ (upper right panel). The interplay of the two antagonist parts of $\theta$ often leads to the occurrence of a local minimum in the coarse-graining of complex 

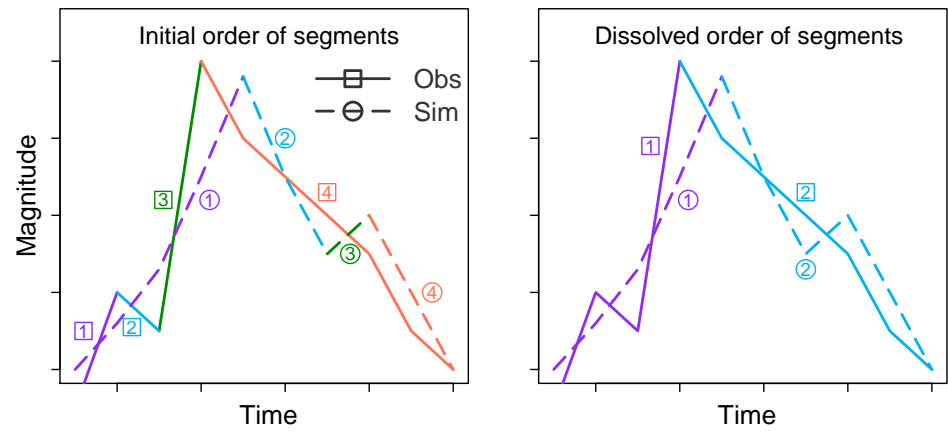

Figure 2. Illustration of the time-ordered matching of segments in the coarse-graining procedure. The rising and falling segments of the simulation (sim) and observation (obs) are numbered and colour-coded according to their chronological order. Series distance compares segments with identical number and/or colour.
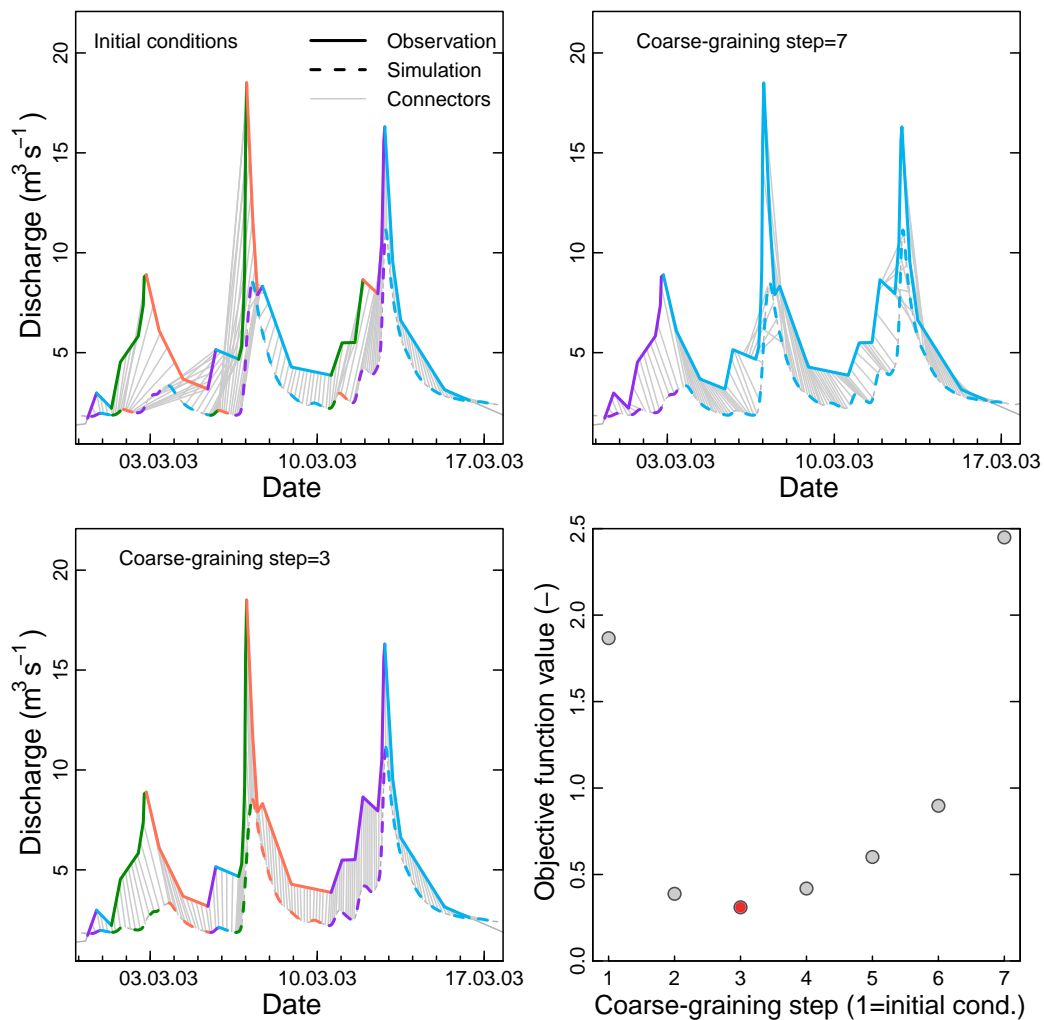

Figure 3. Coarse-graining steps: all plots contain data from the same multi-peak discharge event but for different levels of coarse-graining. The initial conditions (top left panel) are characterized by a large number of poorly matching simulated (dashed) and observed (solid) segments as indicated by the non-intuitively placed SD connectors (grey lines). Segments required to match according to the chronological order constraint of SD are indicated by matching colours. In the last coarse-graining step (top right panel) the connectors are placed more meaningfully but the representation of the entire event by only two segments (one rise, one fall) appears inadequately coarse. The optimal level of coarse-graining, here reached at step three, yields visually acceptable connectors while preserving a detailed segment structure (bottom left panel). This step is associated with a minimum of the coarse-graining objective function (Eq. 2), indicated by the red dot in the bottom right panel. Grey dots indicated the values of the objective function for all other coarse-graining steps.

multi-peak events. The related reduction step can then be regarded as the optimal degree of coarse-graining and the final values of SD time and magnitude errors are determined based on this level. In "simple" events in which no or little coarse graining is required, the ob- jective function values often increase fairly linearly. In any case SD time and magnitude errors are determined based upon the coarse-graining step with the smallest $\theta$ value. 


\subsection{Modifications in the SD error model}

In the initial version of $\mathrm{SD}$, the magnitude error $\left(E_{\mathrm{SD}, Q}\right)$ was calculated as the absolute difference between points in sim and obs linked by a Series Distance connector $(c)$ :

$E_{\mathrm{SD}, Q}(c)=Q_{\mathrm{obs}}(c)-Q_{\mathrm{sim}}(c)$.

In the current version, the magnitude error can alternatively be scaled by the mean of the connected points:

$E_{\mathrm{SD}, Q}^{*}(c)=\frac{Q_{\mathrm{obs}}(c)-Q_{\mathrm{sim}}(c)}{\frac{1}{2}\left(Q_{\mathrm{obs}}(c)+Q_{\mathrm{sim}}(c)\right)}$.

This yields a relative and hence dimensionless expression of the vertical error $\left(E_{\mathrm{SD}, Q}^{*}\right)$, which facilitates the construction of uncertainty ranges of variable width (see Sect. 3). As in the first version of SD, both absolute and relative vertical error values $E_{\mathrm{SD}, Q}^{(*)}>0$ indicate that $Q_{\mathrm{obs}}(c)>Q_{\mathrm{sim}}(c)$. The calculation of Series Distance timing errors $\left(E_{\mathrm{SD}, t}\right)$ according to Eq. (5) remained unchanged. Error values of $E_{\mathrm{SD}, t}>0$ indicate that obs occurs later than sim:

$E_{\mathrm{SD}, t}(c)=t_{\mathrm{obs}}(c)-t_{\mathrm{sim}}(c)$.

Similar to the scaling of the vertical error, the timing error could also be scaled using, e.g., event duration. This could be helpful if the error compared to the length of the event or the average length of all events in the time series is of interest.

The application of SD timing and magnitude error models $\left(E_{\mathrm{SD}, t}(c)\right.$ and $E_{\mathrm{SD}, Q}(c)$ ) makes sense where timing errors are both present and detectable, i.e. during events in which discharge is not constant in time. During low-flow conditions time offsets are, however, difficult, if not impossible to detect. Therefore, a simple one-dimensional, vertical, "standard" error model analogous to Eq. (3), which relates values at the same time step $t$, suffices here:

$E_{\mathrm{S}}(t)=Q_{\mathrm{obs}}(t)-Q_{\mathrm{sim}}(t)$.

Analogously to the scaled vertical SD error model in Eq. (4), a scaled version of the one-dimensional vertical error model $\left(E_{\mathrm{S}}^{*}(t)\right)$ was added:

$E_{\mathrm{S}}^{*}(t)=\frac{Q_{\mathrm{obs}}(t)-Q_{\mathrm{sim}}(t)}{\frac{1}{2}\left(Q_{\mathrm{obs}}(t)+Q_{\mathrm{sim}}(t)\right)}$.

\section{Error dressing: a heuristic approach for the construction of uncertainty ranges}

The SD concept can be applied to a variety of tasks such as model diagnostics, parameter estimation, calibration, or the construction of uncertainty ranges. In this section we provide one example thereof and describe a heuristic approach for the construction of uncertainty ranges for deterministic streamflow simulations. Uncertainty ranges provide regions of confidence around an uncertain estimate and are of practical relevance and a straightforward means of highlighting and of assessing magnitude and timing uncertainties of hydrological simulations or forecasts. Conceptually, uncertainty ranges should be wide enough to capture a significant portion of the observed values but as narrow as possible to be precise and, thus, meaningful. These requirements are antagonistic as large uncertainty ranges, which capture most or all observations, are usually imprecise to a degree that makes them useless for decision-making purposes (Franz and Hogue, 2011).

The method we propose here follows the concept proposed by Roulston and Smith (2003) and yields quantitative estimates of forecast uncertainty by "dressing" single forecasts with historical error statistics. The original approach was designed to dress ensemble forecasts; for SD it was adapted to deterministic streamflow simulations and extended from one dimension (magnitude) to two (magnitude and timing). Like statistical approaches to uncertainty assessment, error dressing is based on the fundamental assumptions of ergodicity and stationarity, i.e. the assumption that errors that occurred in the past are reliable predictors for errors in the future. In the following we first outline the regular, one-dimensional deterministic error dressing method and then describe its modifications for SD.

\subsection{The one-dimensional case}

Provided with a record of past streamflow observations $\left(O_{\text {hist }}\right)$ and corresponding model simulations $\left(S_{\text {hist }}\right)$, any valid error model such as Eq. (6) can be applied to calculate a distribution of historic errors. This distribution can then be sampled (Fig. 4, upper left panel) using a suitable strategy and the selected subset of errors can be applied to each time step of the simulation. Connecting all upper and all lower values of the dressed errors yields corresponding envelope curves (Fig. 4, upper right panel). For this procedure Roulston and Smith (2003) coined the term error dressing.

The choice of the sampling strategy, however, strongly influences the statistics of the resulting uncertainty ranges and should be carefully selected. In our case, the precondition was that the approach should be extendible to twodimensional cases to allow its later application to the error distributions of the SD approach. Therefore, we defined the sampling strategy according to the variance contribution, which is straightforward to apply for the one-dimensional case: for each point of the error distribution its relative contribution $\left(\mathrm{d} \sigma_{i}^{2}\right)$ to the unbiased variance of the total error distribution $\left(\sigma_{x}^{2}\right)$ is calculated according to Eq. (8):

$\mathrm{d} \sigma_{i}^{2}=\frac{\left(x_{i}-\bar{x}\right)^{2}}{n \sigma_{x}^{2}} 100$.

Here $\bar{x}$ and $n$ denote the mean and the size of the corresponding error distribution. The usage of the unbiased variance, having $n$ in the denominator not $n-1$, ensures that all $\mathrm{d} \sigma_{i}^{2}$ sum up to 100 . Next, all points of the error distribution are 
ordered by the values of $\mathrm{d} \sigma_{i}^{2}$, and, starting with the smallest, a desired subset of all $\mathrm{d} \sigma_{i}^{2}$, e.g. $80 \%$ is taken from the list. This subset represents an informal probability $\left(p \in\left[\begin{array}{ll}0 & 1\end{array}\right]\right)$ as it relates to the number of observations that fall within the uncertainty range. Small values of $p$ are associated with narrow (sharp) uncertainty ranges but at the cost of a higher portion of true values that fall outside. Contrary, high values of $p$ cause wide (imprecise) uncertainty ranges which, however, contain most errors that occurred in the past. For practical applications, typically coverages of 80 to $90 \%$ are chosen. In Fig. 4, top left panel, the coverage was set to $p=0.8$.

\subsection{The two-dimensional case}

SD yields two-dimensional distributions of coupled errors in timing and magnitude and thus requires a two-dimensional strategy for the sampling of error subsets and the construction of envelope curves (Fig. 4, lower row panels).

How does one sample from bivariate distributions of coupled errors with different units? Statistics and computational geometry offer concepts based on the ordering of multivariate data sets, such as geometric median or centre point approaches. The former provides a central tendency for higher dimensions and is a generalization of the median which, for one-dimensional data, has the property of minimizing the sum of distances. Centre points are generalizations of the median in higher-dimensional Euclidean space and can be approximated by techniques such as the Tukey depth (Tukey, 1975) or other methods of depth statistics (Mosler, 2013). Here, however, we want the errors to be centred around the mean (not around the median). Hence, we apply the same concept that we use for the one-dimensional case to SD in that we sample based on the combined contribution of each point to the total variance. Analogously to Eq. (8) we calculate the relative timing $\left(\mathrm{d} \sigma_{t}^{2}\right)$ and magnitude $\left(\mathrm{d} \sigma_{Q}^{2}\right)$ contribution of each point to the total variances of the corresponding distributions. Their sum yields an estimate of the combined contribution of each point to the combined variance of both error distributions:

$\mathrm{d} \sigma_{t+Q}^{2}=\mathrm{d} \sigma_{t}^{2}+\mathrm{d} \sigma_{Q}^{2}$.

Analogously to the one-dimensional case, the points are ordered by increasing combined variance contribution $\mathrm{d} \sigma_{t+Q}^{2}$, and, starting from the point with the smallest value which is close to or at the mean, a subset of errors can be extracted. The shape of the resulting subset depends on the underlying distribution of errors. Uncorrelated errors yield more or less circular or oval shapes (Fig. 4, lower left panel). By contrast, correlated errors yield different shapes, which is valuable for diagnostic purposes.

SD distinguishes periods of low flow, rising, and falling limbs. Hence, subsets of two 2-D error distributions (rising and falling limb) and from one one-dimensional error distribution (low flow) are calculated and applied to each point of
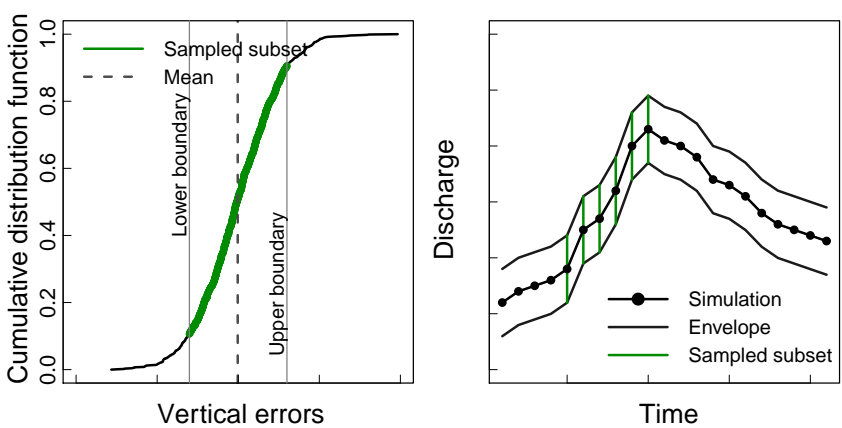

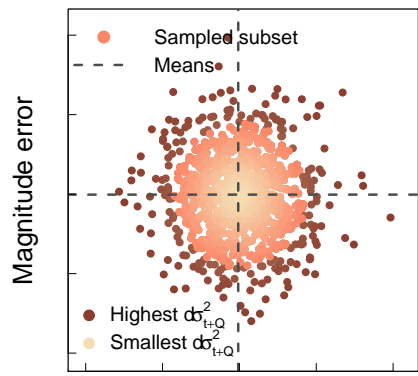

Timing error

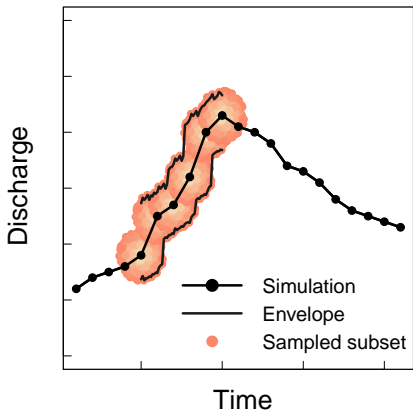

Figure 4. Sketch of the one- and two-dimensional error dressing method using normally distributed random numbers $(n=1000)$. The upper row panels show the one-dimensional case with an empirical cumulative distribution function of errors (upper left panel) and an $80 \%$ subset thereof sampled according to increasing variance contribution. The application (dressing) of the subset of errors to a hydrograph and the construction of the corresponding envelop curves is illustrated in the upper right panel. The lower row panels show the same procedure for the two-dimensional case. From the two-dimensional distribution of empirical errors (bottom left panel) $80 \%$ (colour-coded) are again sampled according to the combined variance contribution of both distributions (colour ramp). The bottom right panel contains a sketch of the two-dimensional error dressing method and the construction of envelope curves. Please note that the use of normally distributed numbers yields symmetrical samples and envelopes, which is usually not the case for realworld data, which are usually skewed.

a simulation: points of low flow are dressed with the lowflow error subset, points of rise with error subsets from rising limbs, etc. Altogether this yields a region of overlapping error ovals around a simulation (Fig. 4, lower right panel), which can for convenience be represented by an upper and lower envelope curve. These lines are found by subdividing the time series into time slices of length $\mathrm{d} t$ (the temporal resolution of the original series), centred around each edge node of series. In each time slice, the magnitude and timing of the largest and smallest error are identified. These values span the upper and lower limit of the uncertainty envelope, respectively. Using linear interpolation yields the upper and lower limits of the envelope at the points in time of the original series, which is useful to calculate evaluation statistics. 


\section{Case study}

This case study, based on real-world data, serves to present and to discuss relevant aspects of SD by comparison with a benchmark error model (BM).

\subsection{Data and site properties}

We used discharge observations $\left(O_{\text {hist }}\right)$ of a 6-year period (30 October 1999-30 October 2005) from gauge "Hoher Steg" (HOST), which is located in the small alpine catchment of the Dornbirner Ach River in north-western Austria. Catchment size is $113 \mathrm{~km}^{2}$, the elevation range is 400-2000 m a.s.l., and mean annual rainfall differs between 1100 and $2100 \mathrm{~mm} \mathrm{yr}^{-1}$. For the 6-year period, hourly hydrometeorological time series $(n=52633$ time steps) were used to drive an existing, calibrated conceptual water budget model of the type LARSIM (Large Area Runoff Simulation Model, gridded version, resolution $=1 \mathrm{~km}^{2}$; Ludwig and Bremicker, 2006), which yielded acceptable simulations $\left(S_{\text {hist }}\right)$ with a NASH of 0.78 . Please note that for the discussion of the SD concept, neither the model itself nor the catchment properties are particularly relevant. The main purpose of the case study was to apply realistic data. This is also the reason why we used the entire 6-year period to both derive and apply the error distributions; i.e. we did not distinguish periods of error analysis and error application.

\subsection{Conceptual setup}

For the benchmark model, we derived distributions of 1-D vertical errors. We did not differentiate cases of low flow and events, which is rather simplistic but standard practice. For the SD approach we did differentiate these cases. This may be considered an unfair advantage for SD as it allows the construction of more custom-tailored uncertainty envelopes. However, as the objective of the case study is not a competition between the two approaches but a way to present interesting aspects of SD, we considered it justified. For SD, the required starting and end points of hydrological events were manually determined both in $O_{\text {hist }}$ and $S_{\text {hist }}$ by visual inspection. Altogether there were $n=123$ events in each series, and they were fully matching; i.e. no missing events or false alarms occurred. The resulting contingency table is obviously trivial and therefore not discussed further here.

Both for $\mathrm{SD}$ and $\mathrm{BM}$, we applied scaled errors $\left(E_{\mathrm{SD}, Q}^{*}(c)\right.$ according to Eq. (4) and $E_{\mathrm{BM}}$ according to Eq. (7), respectively), as we found that compared to the standard error model, they are more applicable across the usually large discharge ranges present in hydrographs. For SD, the weights $\gamma_{1}, \ldots, \gamma_{4}$ used in the objective function of the coarsegraining procedure (Eq. 2) were set to $\frac{1}{7}, \frac{1}{7}, \frac{5}{7}$, and 0 , respectively, based on iteratively maximizing the visual agreement of segments in matching events of sim and obs. Additional studies with different data sets (not shown here) yielded sim- ilar optimal weights, which corroborates that this is a relatively robust choice and sufficient for a proof of concept, as intended in this study. For more widespread applications, a detailed sensitivity analysis is desirable. Such an analysis is, however, difficult as several different time series, flow conditions, and rainfall-runoff events would have to be visualized and compared. Moreover, there is no robust benchmark available to which we may compare the outcome of the proposed coarse-graining procedure. For this reason we provide software such that any interested person can find out for him/herself whether the proposed method suits his or her needs or not.

Based upon SD and BM we derived empirical error distributions from the entire test period and then used them, in the same period, to construct uncertainty envelopes around the simulation $S_{\text {hist }}$ using the error dressing approach as described in Sect. 3. To ensure comparability we enforced identical coverages for both approaches during the construction of the envelope curves; i.e. we made sure that the desired fraction of observations (e.g. $80 \%$ ) fell within the uncertainty envelope. For the standard error model this was straightforward: if from the 1-D distribution of errors a subset of $p=80 \%$ is selected and used to construct the uncertainty envelope as described in Sect. 3.1 for the same period of time, then by definition the number of observations within the envelope must also be $80 \%$. For SD, however, as a consequence of error ovals overlapping in time (Fig. 4, lower right panel), this is not self-evident and typically many more observations fall within the uncertainty envelope than the level $p$ at which the subset of the 2-D error distribution is sampled. This issue was solved by iteratively sampling the error distributions at various levels of $p$ until the desired percentage of observations (here: $80 \%$ ) fell within the uncertain envelope.

\subsection{Evaluation of deterministic uncertainty ranges}

The evaluation of deterministic uncertainty ranges requires methods to quantify properties such as coverage or precision. Here we propose a set of statistics which can be applied to uncertainty ranges irrespective of how they were constructed. While this ensures comparability of the SD and BM-derived ranges, it does not exploit the advantages of the SD approach, i.e. separate treatment of time and magnitude uncertainties.

1. Coverage $(\phi)$ is the most intuitive criterion. It quantifies the ratio of observations that fall inside the simulated uncertainty range and can take values between 0 (no single observed value included) and 1 (all observations included). $\phi$ can easily be obtained as the number of observations ( $n_{\text {obs }}$ ) that fall inside the uncertainty range around a simulation, divided by the total length of the time series $(n)$ :

$\phi=\frac{n_{\mathrm{obs}}}{n}$. 
2. Precision (PRC) allows the comparison of different uncertainty ranges. PRC is the average width of the uncertainty envelope, i.e. the average difference of the upper $\left(\mathrm{UE}^{+}(t)\right)$ and the lower $\left(\mathrm{UE}^{-}(t)\right)$ envelope curve. The smaller PRC, the sharper the uncertainty range. High coverages $(\phi)$ typically require wide uncertainty ranges and, thus, high values of PRC. PRC has the same unit as the discharge time series.

$$
\mathrm{PRC}=\frac{1}{n}\left(\mathrm{UE}^{+}(t)-\mathrm{UE}^{-}(t)\right)
$$

3. Finally we suggest scaling PRC by the value of the simulation according to Eq. (4), i.e. to express uncertainty relative to the magnitude of the simulation. $\mathrm{PRC}^{*}$ is dimensionless and decreases with decreasing width of the uncertainty range. An uncertainty range of zero width yields a PRC* of 0 . Hence, small values of PRC* indicate high skill.

$$
\mathrm{PRC}^{*}=\frac{1}{n} \frac{\left(\mathrm{UE}^{+}(t)-\mathrm{UE}^{-}(t)\right)}{Q_{\operatorname{sim}}(t)}
$$

In the case study, we used $\phi$ as a means to ensure comparability rather than for comparison: coverage for both the SD and BM approach was set to $80 \pm 0.5 \%$. For SD the required percentage of sampled errors was found by trial and error to be $p=76 \%$ (Table 3). With coverage equalized, SD and BM can be directly compared by PRC and PRC*. High (relative) precision, i.e. small values of $\mathrm{PRC}^{(*)}$, indicate better performance. If the evaluation of uncertainty ranges with respect to over- and undershooting is of interest, additionally the percentage of observations above or below the uncertainty range can be computed analogously to Eq. (10). This is for instance of interest for flood forecasters who try to minimize overshooting or water supply managers who try to minimize undershooting. For the sake of brevity, this has not been further considered here.

\section{Results and discussion}

In this section we first discuss some general aspects of the SD concept and then compare it to the benchmark approach using the case study data.

\subsection{Potential and limitations of the core SD concept}

Series Distance is an elaborate method for the comparison of simulated and observed streamflow time series. The concept allows the distinction between different hydrological conditions (low flow and rising and falling limbs) and determines joint errors in timing and magnitude of matching points within matching segments of related hydrographs. Differences in the high- and/or low-frequency agreement of the obs and sim hydrographs are considered with an iterative coarse-graining procedure, which effectively mimics visual hydrograph comparison. This differentiated evaluation makes SD a powerful tool for model diagnostics and performance evaluation.

The challenges of SD are, however, in the details: the robust, precise, and meaningful partitioning of the hydrograph into periods of low flow and events is difficult. We tested various approaches including baseflow separation and filtering techniques (e.g. Douglas and Peucker, 1973; Chapman, 1999; Perng et al., 2000; Eckhardt, 2005), penalty functions (Drabek, 2010), fuzzy logic (Seibert and Ehret, 2012), and the methods proposed by Merz and Blöschl (2009) and Norbiato et al. (2009). In all cases, the results were unsatisfactory when applied to a range of different flow regimes. The same applies for the matching of conjugate events in obs and sim. Currently, there is no robust and automated method available for any of the two cases. Possible remedies are the adaptation of any of the methods proposed above to specific conditions (Seibert et al., 2016), manual event detection, and matching, or one could treat the entire time series as a single, long event, at the expense of losing the separate treatment of low-flow cases. Within an event, the quality of the segment matching significantly determines the quality of the subsequent matching of obs and sim points and hence the quality of the SD error calculation. This challenge has been solved in a mostly very satisfactory way by the iterative coarse-graining procedure. The resulting set of matching segments and the required degree of coarse-graining is in itself a useful result which can be used for comparative hydrograph analysis.

Qualitative analyses of the weighting factors $\gamma_{1} \ldots \gamma_{4}$ in Eq. (2) confirmed that these parameters emphasize different aspects of the hydrograph and thus allow for a flexible adaptation of the pattern matching procedure to different flow regimes. Applied to a single event, different combinations of $\gamma$-parameters cause different segments to be identified and matched, leading to differing SD results and aggregation steps. Overall, $\gamma_{1}$ and $\gamma_{2}$ are less sensitive than $\gamma_{3}$ and $\gamma_{4}$. Table 1 qualitatively summarizes the impact of the different weighting factors. Figure 5 provides the coarse-graining solution for the event depicted in Fig. 3 if $\theta$ is parametrized using equal weights (case no. 5 in Table 1). This plot highlights that different solutions can be acceptable and that coarsegraining remains to a certain degree arbitrary. In any case the parametrization of $\theta$ requires a visual verification as small modifications may yield different results. We found that the configuration presented in the case study (Sect. 4.2) which punishes large timing errors $\left(E_{\mathrm{SD}, t}^{*}\right)$ produces good agreement with visual coarse-graining for different events or conditions and we thus suggest it as default parametrization. A more in-depth study of the impacts of $\gamma_{1} \ldots \gamma_{4}$ using streamflow data from different regimes and events would, however, be desirable.

The hydrograph matching algorithm (HMA) proposed by Ewen (2011) is, to our knowledge, the only method which 
Table 1. Qualitative description of the impact of the different weighting factors of the objective function $\theta$ (Eq. 2), which governs the coarsegraining procedure. Note: none of the extreme parametrizations described by the cases nos. 1-4 is meaningful as any of them prevent the comparison of hydrologically similar points.

\begin{tabular}{lccccl}
\hline Case & $\gamma_{1}$ & $\gamma_{2}$ & $\gamma_{3}$ & $\gamma_{4}$ & Impact \\
\hline 1 & 1 & 0 & 0 & 0 & no aggregation of segments \\
2 & 0 & 1 & 0 & 0 & no aggregation of segments \\
3 & 0 & 0 & 1 & 0 & horizontal differences are minimized, i.e. vertical comparison \\
4 & 0 & 0 & 0 & 1 & vertical differences are minimized, i.e. horizontal comparison \\
5 & $\frac{1}{4}$ & $\frac{1}{4}$ & $\frac{1}{4}$ & $\frac{1}{4}$ & equal weights, compare Fig. 5 \\
6 & $\frac{1}{7}$ & $\frac{1}{7}$ & $\frac{5}{7}$ & 0 & suggested default, compare Fig. 3 (bottom left panel) \\
\hline
\end{tabular}

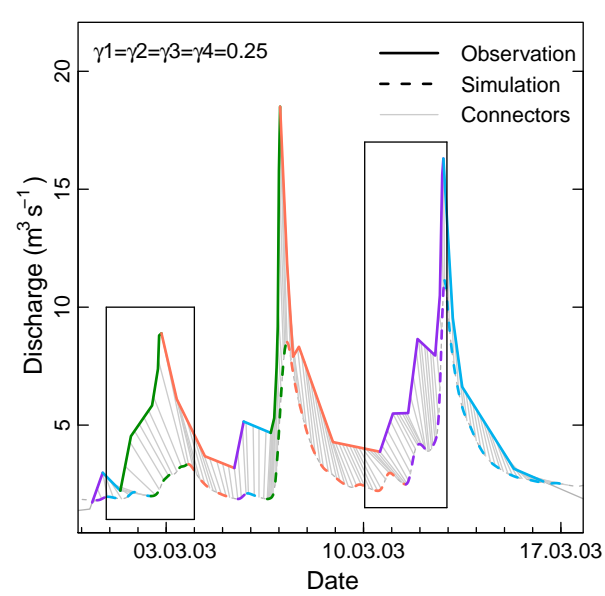

Figure 5. Optimal coarse-graining solution of the event depicted in Fig. 3 if equal weights ( $\gamma$ parameters) are applied to the objective function $\theta$ (Eq. 2). In this case the coarse-graining procedure selects different edge nodes for two segments (see black boxes) leading to slightly larger timing and smaller magnitude errors compared to the bottom left panel in Fig. 3.

is similar to the SD concept in the sense that it relates elements of an observed to elements in a simulated hydrograph in an intuitive manner. Similar to SD, the HMA uses connectors ("rays") to establish these relationships. However, the manner in which these connectors are identified is different. The HMA moves chronologically through all elements of obs and calculates the distance to points in sim which are located within a defined window around the element in obs using a penalty function. This procedure generates a (possibly huge) matrix of penalty values. In a second step the optimal "path" through this matrix is identified, which yields the connectors. This makes the HMA computationally demanding. However, the same also applies for SD as the coarse-graining scheme may require a large number of iterations. The advantage of $\mathrm{SD}$ is that unique relationships of points in obs and sim are established, which is not the case for HMA. Leaving aside these methodological finesses, we believe that for hydrological studies there is a large potential for "intuitive" distance metrics which is not yet fully exploited: in the intercompar- ison study of Crochemore et al. (2014) both HMA and SD closely resembled expert judgement and outperformed standard (vertical) distance metrics during high- and, for HMA, also low-flow conditions.

\subsection{Potential and limitations of the error dressing method}

Error dressing is a simple method and straightforward to apply. Conceptually it is very similar to statistical concepts like the total uncertainty method introduced by Montanari and Grossi (2008) insofar as it does not distinguish between different sources of uncertainty. Unlike rigorous statistical concepts, error dressing, however, does not make any assumptions regarding the nature of the population of errors: they are directly sampled from the empirical distribution, thus avoiding the need to fit a theoretical distribution to the data. The fundamental assumption of error dressing is hence that the available sample represents the population and implies that the skill of the resulting uncertainty ranges strongly depends on the representativeness of the empirical distribution of errors. This may not be the case if records are short and/or if the available data only cover a limited range of conditions. This is, however, a frequent problem of statistical methods for uncertainty assessment (not only in hydrology), where often the extremes are of interest, although they are rare by definition (Montanari and Grossi, 2008). Further uncertainties arise from erroneous observations, which is a common problem in hydrology. These conceptual limitations lead to the fundamental question of whether it is better to profit from statistical (or heuristic) information on the basis of the stationarity assumption or to neglect it by questioning the assumption itself (Montanari, 2007). This discussion is, however, beyond the scope of this study.

The error dressing concept in the presented form does not distinguish between seasonality or different flow magnitudes as the same error distributions are applied to each rising (and/or falling) limb. More sophisticated implementations are of course possible, such as a differentiation of errors according to flow magnitudes to better capture extremes, or differentiation according to forecast lead times. 
The same applies for the sampling strategy: as an alternative to the method presented here based on combined variance contribution, the sampling of specific quantiles using the median as central reference or the fitting and application of any parametric function to the distribution is of course possible. A practical insight from applying the error dressing concept is that the variance-based method effectively filters outliers, which sometimes occur when errors are calculated between poorly matching segments.

A last general issue relates to the sampling from the twodimensional error distribution. Due to the superposition of error clouds in successive time steps it is possible that errors in timing at one time step mimic errors in magnitude at neighbouring time steps (Fig. 4, bottom right panel). This depends on the temporal extent of the error ovals. As a consequence, the relationship between $p$, which defines the size of the subset from the distribution, and coverage $(\phi)$ becomes non-unique. In any case it is not directly linear as in the onedimensional case in which $p$ equals $\phi$ per definition (at least for the period of calibration). Typically $\phi$ exceeds $p$ in the two-dimensional case, and desired coverage rates of $\approx 80 \%$ require us to set $p$ to $\approx 0.65-0.75$. If a specific coverage is desired, the related value of $p$ is best found by iteration. Altogether, the error dressing concept seems suitable for practical applications where long time series are available but more sophisticated uncertainty assessments are not feasible, either because of the required effort or because of limited knowledge of the underlying system.

\subsection{Case study results}

As described in Sect. 4.2, within the 6-year time series, altogether $n=123$ events were manually identified in both obs and sim. The events matched perfectly; i.e. no missed events or false alarms occurred. This is often the case for simulations of responsive catchments where rainfall events trigger runoff events in most cases and where the precipitation time series thus carries important information about the occurrence of hydrological events. This is not necessarily the case for hydrological forecasts, especially mid- to long-term, where false precipitation events can generate false hydrological events. In the latter case, event-based information contained in the contingency table can be valuable. The mean event durations were 146 and $154 \mathrm{~h}$ for obs and sim, respectively, and on average each event initially contained 13 (sub)peaks. The optimal level of event comparison was on average achieved after two coarse-graining steps, which reduced the number of peaks on average to four and led to average durations of $37 \mathrm{~h}$ for rising limbs and $109 \mathrm{~h}$ for falling limbs for both obs and sim. These statistics again bear diagnostic potential as they can be interpreted as surrogates for the mean concentration time of the catchment or as a reservoir constant and can thus be compared to other data. Generally, the matching of segments resulting from the coarse-graining procedure corresponded well with visual hu- man reasoning (not shown). In the following we compare the error distributions and uncertainty envelopes derived from the SD and BM approach for our test case.

\subsubsection{Comparison of error distributions}

Altogether four error distributions were calculated: for SD two 2-D distributions (one for the rising and one for the falling event limbs) and one 1-D distribution for the low-flow conditions; for BM a single 1-D distribution of magnitude errors for the entire time series. The distributions are shown in Fig. 6, corresponding statistics in Table 2.

Comparing the 2-D distributions reveals distinct differences in shape: for the rising limbs the distribution is rather oval; for the falling limbs it is almost circular. This is particularly evident in the sampled subsets. The uniform spread of the errors within the oval and the circle indicates that for the data at hand, the timing and magnitude errors are largely uncorrelated but dependent upon the hydrological conditions (rise or fall). The (scaled) magnitude errors for both distributions are located between \pm 1.5 . The magnitude biases for both distributions are relatively small and lie, according to the ranges provided by Di Baldassarre and Montanari (2009), within the error of measurement: $\mathrm{SD}_{Q \text {,rise }}=0.1$ for the rising limbs, $\mathrm{SD}_{Q \text {,fall }}=0.008$ for the falling limbs. Note that positive magnitude biases indicate simulations that on average underestimate the observations. For timing errors, the differences are more pronounced: while for the rising limbs, timing errors are located between $\pm 10 \mathrm{~h}$ for the sampled subset and biased by $-0.2 \mathrm{~h}$ (indicating simulations lagging behind the observations), for the falling limbs both the bias $(-3 \mathrm{~h})$ and the range $( \pm 20 \mathrm{~h})$ are much larger. Please note that we discuss the timing errors of the subset here rather than those of the entire sample, as the latter include few but large outliers caused by occasional poor matching of falling limbs during coarse-graining.

Together, these results confirm that different flow conditions, i.e. low-flow, rising or falling limbs of events, exhibit different error characteristics. This suggests that a differentiation between hydrological conditions can be meaningful. For instance, timing errors of the recession in the case study would be strongly underestimated by timing errors of the rising limbs, and vice versa, as depicted in the lower panel of Fig. 8. The comparison of 1-D distributions of the SD and BM model revealed that important error characteristics of rare events can be shadowed by frequent but often less important low-flow conditions.

\subsubsection{Comparison of uncertainty envelopes}

Subsets of both the SD and BM error distributions were used to construct uncertainty envelopes (UE) around the entire simulated time series $S_{\text {hist }}$. For better visibility of the details, only a 3-week period is shown in Fig. 7; the envelope statistics presented in Table 3, however, are based on the entire se- 
Table 2. Statistical properties of the individual Series Distance (SD) and benchmark (BM) error distributions from the case study. For the entire distribution we provide the first and third quartile, the mean, median, and the percentage of outliers (data points which are more than 3 standard deviations apart from the mean). For the subset we provide the sampled upper (maximum) and lower (minimum) boundaries. The subscripts with SD refer to errors in magnitude $(Q)$ and timing $(t)$ separately for the rising (rise) and falling (fall) limbs, respectively. $\mathrm{SD}_{\mathrm{LF}}$ provides results for the periods of low flow.

\begin{tabular}{|c|c|c|c|c|c|c|c|}
\hline \multirow{2}{*}{$\begin{array}{l}\text { Error } \\
\text { Distribution }\end{array}$} & \multicolumn{5}{|c|}{ Entire distribution } & \multicolumn{2}{|c|}{ Sampled subset } \\
\hline & $25 \%$ quartile & Mean & Median & $75 \%$ quartile & $\%$ outlier & Minimum & Maximum \\
\hline $\mathrm{SD}_{Q, \text { rise }}(-)$ & -0.15 & 0.11 & 0.13 & 0.39 & 0.7 & -0.44 & 0.67 \\
\hline $\mathrm{SD}_{Q, \text { fall }}(-)$ & -0.23 & 0.01 & 0.01 & 0.25 & 0.5 & -0.54 & 0.55 \\
\hline $\mathrm{SD}_{t, \text { rise }}(\mathrm{h})$ & -0.50 & -0.22 & 0.66 & 1.60 & 2.1 & -8.41 & 7.98 \\
\hline $\mathrm{SD}_{t, \text { fall }}(\mathrm{h})$ & -3.89 & -2.87 & 0 & 1.56 & 2.9 & -21.61 & 15.86 \\
\hline $\mathrm{SD}_{\mathrm{LF}}(-)$ & -0.64 & -0.35 & -0.37 & -0.06 & 0.1 & -0.89 & 0.19 \\
\hline $\mathrm{BM}(-)$ & -0.54 & -0.23 & -0.24 & 0.09 & 0.1 & -0.83 & 0.37 \\
\hline
\end{tabular}
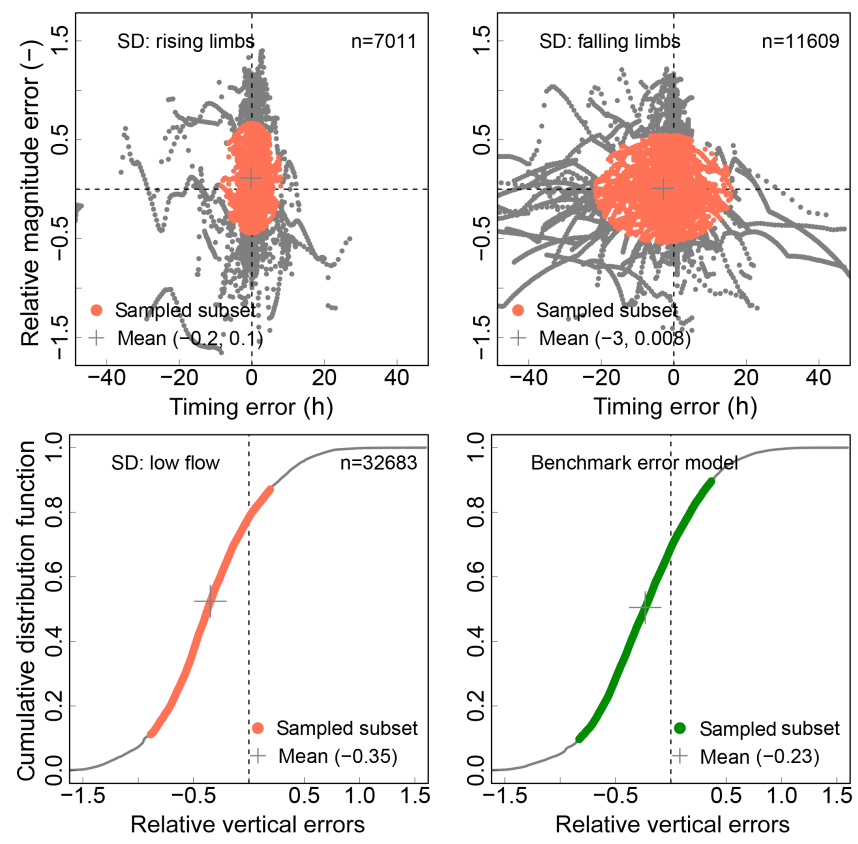

Figure 6. One- and two-dimensional error distributions from the case study. The upper row contains Series Distance (SD) results for the rising and falling limbs. The left panel in the lower row shows the one-dimensional SD distribution of errors for the periods of low flow. The panel in the bottom right contains the 1-D distribution of magnitude errors of the benchmark model (BM) for the entire time series. The highlighted subset represents the $80 \%$ subset used to construct the uncertainty envelopes. Distribution statistics are provided in Table 2. To improve the readability of the upper two panels, we restricted their timing axes to the range [ -4545$]$. The number of outliers, i.e. points outside the range mean $\pm 3 \mathrm{SD}$ (standard deviations) ([-42 36]), was $<1 \%$ for the falling limbs and 1 order of magnitude less for the rising limbs. The dotted lines highlight the origins (all panels). ries. The percentages $p=76 \%$ for SD and $p=80 \%$ for MD of sampled errors in the subsets were selected such that the overall coverage $(\phi)$ of the uncertainty envelopes was $80 \%$ in both cases. Compared to $\mathrm{UE}_{\mathrm{BM}}$, the $\mathrm{UE}_{\mathrm{SD}}$ in Fig. 7 appears both smoother and more inflated. This is due to the timing component of the error model, which spreads the uncertainty envelope in time. This is particularly visible at the beginning of the events. Here, timing errors dressed to a given time step clearly extend to neighbouring time steps, representing the uncertainty about the true event start. In the case of several peaks occurring within a short time (Fig. 7, last event), the smoothing effect of the timing component can lead to a merging of the related uncertainty envelopes towards a single, large region. Also the difference between smaller timing errors in the rising limbs and larger timing errors in the falling limbs are visible. Partly, timing errors of the falling limb even mimic timing errors in the rising limb (compare also Fig. 8, lower panel). The false inflation of the uncertainty envelope due to the timing error is undesirable. The reasons for it are, however, manifold. Possible ways forward to narrow the time-inflated SD uncertainty envelope would be (i) to replace the static timing error model (Eq. (5)) by a relative representation, e.g. by using mean event duration, (ii) to further differentiate the error distributions, e.g. according to flow magnitude and (iii) in the consideration of the autocorrelation of the errors which is typically large in streamflow data. Of course, errors in the coarse-graining can also contribute to false inflation.

In comparison, the uncertainty envelope of the BM model appears slimmer and more precise. However, due to the lack of consideration of timing uncertainties, especially during steep flood rises, the uncertainty envelopes become very narrow. Such a "vanishing" of the uncertainty envelopes implies that there are no timing errors to be expected at all (compare, e.g., the period 6-7 June 2001 in Fig. 7), which is deceptive, keeping in mind the SD results for the timing errors (Fig. 6). We thus consider this aspect a disadvantage of 
Table 3. Coverage $(\phi)$, precision (PRC), and relative precision $\left(\mathrm{PRC}^{*}\right)$ of uncertainty envelopes. $\mathrm{UE}_{\mathrm{SD}}$ and $\mathrm{UE}_{\mathrm{BM}}$ denote Series Distance and benchmark error model, respectively. The last column $(p)$ provides the percentage of sampled values of the corresponding distribution(s).

\begin{tabular}{lcccc}
\hline $\begin{array}{l}\text { Uncertainty } \\
\text { envelope }\end{array}$ & $\begin{array}{c}\phi \\
(-)\end{array}$ & $\begin{array}{c}\text { PRC } \\
\left(\mathrm{m}^{3} \mathrm{~s}^{-1}\right)\end{array}$ & $\begin{array}{c}\text { PRC }^{*} \\
(-)\end{array}$ & $\begin{array}{c}p \\
(\%)\end{array}$ \\
\hline UESD & 80.5 & 8.2 & 1.3 & 76 \\
UEBM & 80.0 & 5.1 & 1.0 & 80 \\
\hline
\end{tabular}

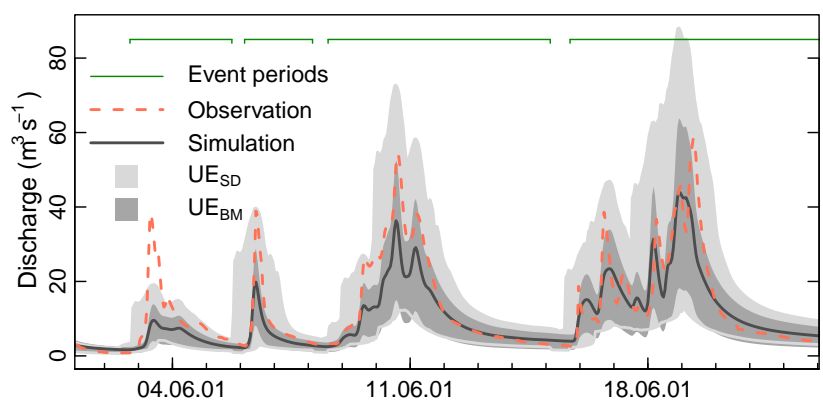

Figure 7. Time series detail showing the resulting one- and twodimensional uncertainty envelopes around the historic streamflow simulation. The envelopes were derived upon Series Distance $\left(\mathrm{UE}_{\mathrm{SD}}\right)$ and the benchmark approach $\left(\mathrm{UE}_{\mathrm{BM}}\right)$, respectively, using error dressing. Please note that the coverage of the SD and BM envelope may differ for different subsets of the time series, like in this detail. For the entire time series, however, the coverage of $\mathrm{BM}$ and SD are identical.

the one-dimensional error dressing method, especially as the timing of flood rises is often critical in hydrological applications (Seibert et al., 2014).

The statistical evaluation of the different uncertainty envelopes (Table 3) confirms the visual impression: the BM uncertainty envelope outperforms SD in terms of absolute and relative precision (PRC and $\mathrm{PRC}^{*}$, respectively) given identical coverage $(\phi)$. On average, $\mathrm{UE}_{\mathrm{SD}}$ is $3.1 \mathrm{~m}^{3} \mathrm{~s}^{-1}$ wider than the benchmark envelope, which corresponds to a relative difference of $30 \%$ as indicated by PRC*. This suggests that the use of the SD concept to construct uncertainty envelopes implies a trade-off between two effects: on the one hand, the explicit consideration of timing errors potentially yields bettertailored uncertainty envelopes, as apparent timing errors can be treated as such. On the other hand, if timing is not a dominant or at least substantial component of the overall error, the time-spreading effect of the SD envelope construction can lead to an undesirable inflation effect. In our case study, the latter effect apparently predominated. For hydrological forecasts based on uncertain meteorological forecasts, however, the opposite may be the case.
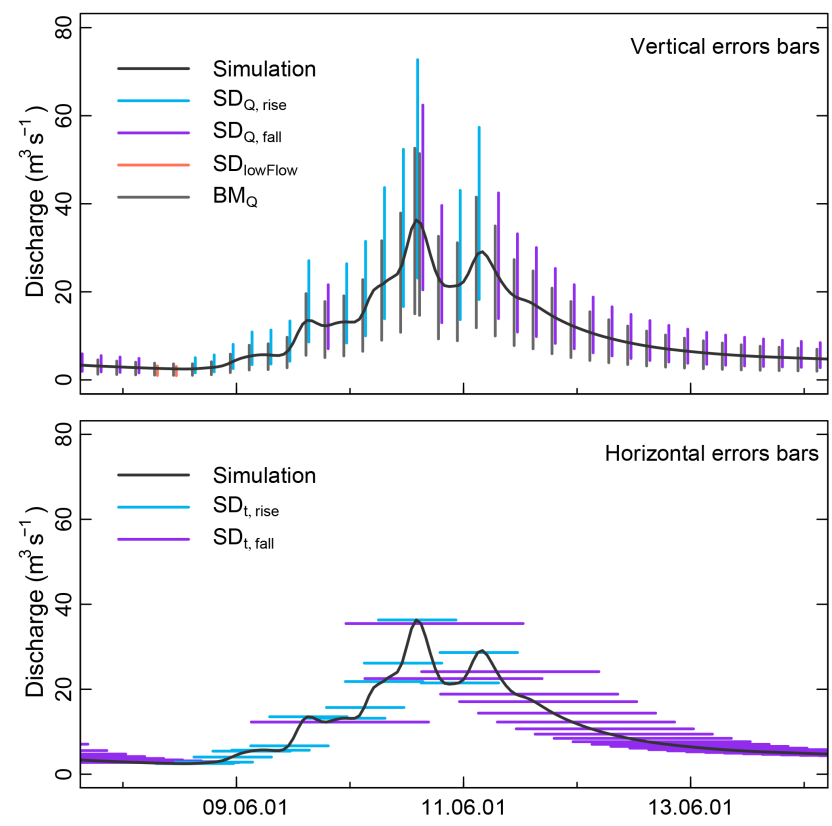

Figure 8. Vertical and horizontal error bars. The upper panel shows magnitude error bars $(Q)$ for the Series Distance (SD) method and the benchmark (BM) approach. For SD different error bars are drawn for low-flow conditions and rising (rise) and falling (fall) limbs. In the BM case the same error bars are applied in all cases. The lower panel shows the corresponding timing error bars $(t)$ of SD (not available for BM), again separately for the rising and falling limbs. To improve readability we plotted error bars only every third hour and introduced a slight time offset between SD and BM (upper panel only). Both panels show a subset of the hydrograph section depicted in Fig. 7 and are based on the same data.

\subsubsection{Disentangling the importance of magnitude and timing errors}

To further investigate the individual effects of errors in timing and magnitude, we also applied them separately to the simulated time series. To this end we applied case-specific subsets of the error distributions - i.e. 2-D errors for rising and falling limbs and 1-D error distributions for low flow - to each point of the simulated time series just as in the previously described error dressing approaches. The difference was that we did not apply the entire error subset (oval or circle) but its projection on the time and magnitude axis, respectively. The resulting uncertainty bars therefore extend from the maximum to the minimum magnitude (upper panel) and timing (lower panel) values of the error subsets and are depicted in Fig. 8. For comparison we also plotted the magnitude errors of the BM approach. In this representation it becomes obvious that the error bars of the SD and BM approach show considerable differences with respect to extent and symmetry. For the magnitude error bars the deviations are most pronounced in the rising limbs and less so in the falling limbs and during low-flow conditions. While the SD 
method reflects the underling characteristics of the errors, the BM method applies the same error to all cases. Constructing an uncertainty envelope from only the SD magnitude errors would yield an envelope comparable to that of BM but be more variable and have higher uncertainty towards overestimations than towards underestimations. Note that the true distribution of errors within the error bars is unknown.

The lower panel in Fig. 8 reveals that the uncertainties with respect to timing are considerable, typically during the recessions. Combining horizontal and vertical errors to construct the 2-D SD uncertainty envelope using the method described in Sect. 3 will inevitably cover a large region. While this is undesirable, it points towards possible alternatives to construct uncertainty ranges: rather than uniting the horizontal and vertical uncertainty components, intersecting them, i.e. to use only elements which are common to both error components,s would also be possible, for example, and most likely narrow the uncertainty envelope. Also, discharge time series usually exhibit considerable autocorrelation and so do related simulation errors. Exploiting this memory effect by time-conditioned sampling of the error distribution via a Markov process would be a further alternative to better tailor uncertainty envelopes (Vrugt et al., 2008; Montanari et al., 1997).

Finally, even if the SD error distributions are not used to construct uncertainty envelopes, knowledge of magnitude and timing error distributions is valuable for model diagnostics: in their approach to identifying characteristic error groups in hydrological time series Reusser et al. (2009) had to inversely infer the effect of timing errors to their signatures; SD offers a method to directly measure timing errors and thus to improve this step.

\section{Conclusions}

The main goal of this paper was to present major developments in the SD concept since its first version presented by Ehret and Zehe (2011). These include the development of an iterative optimization procedure which effectively mimics coarse-graining of hydrographs when comparing them visually. The parameters of the inherent objective function were derived manually for this study; for more widespread applications, however, we recommend an in-depth sensitivity analysis using data from different regimes. Coarse-graining yields a set of matching segments within observed and simulated hydrological time series and the optimal degree of coarse-graining, both of which can be used as input for comparative hydrograph analysis. Further developments include the introduction of a scaled error model, which has proven to be better applicable across large discharge ranges than its non-scaled counterpart, and error dressing, a concept to construct uncertainty ranges around deterministic streamflow simulations or forecasts. Error dressing includes an approach to sample empirical error distributions by increasing vari- ance contribution, which we extended from standard onedimensional distributions to the two-dimensional distributions of combined time and magnitude errors of SD.

Applying the SD concept and a benchmark model (BM) based on standard magnitude errors to a 6-year time series of observations and simulations in a small alpine catchment revealed that different flow conditions (low flow and rising and falling limbs during events) exhibit distinctly different characteristics of timing and magnitude errors with respect to mean and spread. Separate treatment of timing and magnitude errors and a differentiation of flow conditions as done in $\mathrm{SD}$ is thus recommended in general as it preserves useful information. Exploiting these characteristics and their correlations can support targeted model diagnostics. Deeper insights can easily be provided if the error distributions are further differentiated by discharge magnitude classes, by season, or by considering the temporal autocorrelation of errors. The latter would allow the development of a time-conditioned error sampling strategy when constructing 2-D uncertainty envelopes.

Applying the error distributions of both SD and BM to construct uncertainty ranges around the fairly accurate simulation revealed a remarkable timing uncertainty. This suggests that we commonly underestimate the role of horizontal uncertainties in streamflow simulations. For the given data, the BM-derived uncertainty ranges were in consequence visually narrower and statistically superior to the SD ranges. This suggests that the use of the SD concept to construct uncertainty envelopes according to the proposed error dressing method implies a trade-off between two effects: on the one hand, the explicit consideration of timing errors potentially yields better-tailored uncertainty envelopes, as apparent timing errors are treated as such. On the other hand, the timespreading effect of the SD envelope construction, which essentially is the union of the time and magnitude error uncertainty ranges, can lead to an undesirable inflation. For the case study data, the latter effect predominated, while for hydrological forecasts based on uncertain meteorological forecasts the opposite may be the case. This also opens interesting avenues for new ways to construct uncertainty ranges based on the SD concept, e.g. as the intersection (rather than the union) of the two error components.

We conclude that Series Distance is an elaborate concept for the comparison of simulated and observed streamflow time series which can be used both for detailed hydrological analysis and model diagnostics. Its application, however, involves considerably more effort than standard diagnostic measures, which are typically justified if timing errors are dominant or of particular interest. More generally, we believe that for hydrological studies there is a large potential for intuitive distance metrics such as the hydrograph matching algorithm proposed by Ewen (2011) or the SD concept, which should be further exploited as suggested by Crochemore et al. (2014). 
To foster the use of the SD concept and the methods therein we publish a ready-to-use Matlab program code alongside to the manuscript under a Creative Commons license (CC BY-NC-SA 4.0). It is accessible via https://github. com/KIT-HYD/SeriesDistance. This repository also includes extended versions of the SD concept which we did not describe in full length here. These allow for a continuous usage of the method (no data on events required) and/or a differentiation of vertical errors according to flow magnitude.

\section{Data availability}

A development release of the Series Distance program code (Ehret and Seibert, 2016), licensed under a Creative Commons license (CC BY-NC-SA 4.0), is published alongside this manuscript via GitHub https://github.com/KIT-HYD/ SeriesDistance. The repository also includes a sample data set of an observed and a simulated discharge time series.

Parametrization files for the Large Area Runoff Simulation Model (LARSIM, Ludwig and Bremicker, 2006) and the corresponding hydrometeorological data sets which we used in the case study can be obtained from the authors upon request. LARSIM executables can be obtained upon request from the Landesanstalt für Umwelt, Messungen und Naturschutz Baden-Württemberg, Germany (LUBW, 2016).

Acknowledgements. We thank Tilmann Gneiting from the Heidelberg Institute for Theoretical Studies (H-ITS) for valuable discussions on the error dressing concept, Clemens Mathis from Wasserwirtschaft Vorarlberg for providing the case study data and hydrological model, and all users of SD who provided valuable feedback and constructive criticism throughout recent years. We furthermore thank the three anonymous referees for providing valuable comments and acknowledge support for open-access publishing by the Deutsche Forschungsgemeinschaft (DFG) and the Open Access Publishing Fund of Karlsruhe Institute of Technology (KIT).

The article processing charges for this open-access publication were covered by a Research

Centre of the Helmholtz Association.

Edited by: V. Andréassian

Reviewed by: three anonymous referees

\section{References}

Attinger, S.: Generalized Coarse Graining Procedures for Flow in Porous Media, Comput. Geosci., 7, 253-273, doi:10.1023/B:COMG.0000005243.73381.e3, 2003.

Bennett, N. D., Croke, B. F. W., Guariso, G., Guillaume, J. H. A., Hamilton, S. H., Jakeman, A. J., Marsili-Libelli, S., Newham, L. T. H., Norton, J. P., Perrin, C., Pierce, S. A., Robson, B., Seppelt, R., Voinov, A. A., Fath, B. D., and Andreassian, V.: Charac- terising performance of environmental models, Environ. Model Softw., 40, 1-20, doi:10.1016/j.envsoft.2012.09.011, 2013.

Beven, K. and Binley, A.: The future of distributed models: model calibration and uncertainty prediction, Hydrol. Process., 6, 279 298, doi:10.1002/hyp.3360060305, 1992.

Biondi, D., Freni, G., Iacobellis, V., Mascaro, G., and Montanari, A.: Validation of hydrological models: Conceptual basis, methodological approaches and a proposal for a code of practice, Phys. Chem. Earth, 42-44, 70-76, doi:10.1016/j.pce.2011.07.037, 2012.

Blume, T., Zehe, E., and Bronstert, A.: Rainfall-runoff response, event-based runoff coefficients and hydrograph separation, Hydrolog. Sci. J., 52, 843-862, doi:10.1623/hysj.52.5.843, 2007.

Boyle, D. P., Gupta, H. V., and Sorooshian, S.: Toward improved calibration of hydrologic models: Combining the strengths of manual and automatic methods, Water Resour. Res., 36, 36633674, doi:10.1029/2000WR900207, 2000.

Chapman, T.: A comparison of algorithms for stream flow recession and baseflow separation, Hydrol. Process., 13, 701714, doi:10.1002/(SICI)1099-1085(19990415)13:5<701::AIDHYP774>3.0.CO;2-2, 1999.

Cloke, H. L. and Pappenberger, F.: Evaluating forecasts of extreme events for hydrological applications: An approach for screening unfamiliar performance measures, Meteorol. Appl., 15, 181-197, doi:10.1002/met.58, 2008.

Crochemore, L., Perrin, C., Andréassian, V., Ehret, U., Seibert, S. P., Grimaldi, S., Gupta, H., and Paturel, J.-E.: Comparing expert judgement and numerical criteria for hydrograph evaluation, Hydrolog. Sci. J., 60, 402-423, doi:10.1080/02626667.2014.903331, 2014.

Dawson, C. W., Abrahart, R. J., and See, L. M.: HydroTest: A web-based toolbox of evaluation metrics for the standardised assessment of hydrological forecasts, Environ. Model. Softw., 22, 1034-1052, doi:10.1016/j.envsoft.2006.06.008, 2007.

De Lannoy, G. J. M., Houser, P. R., Pauwels, V. R. N., and Verhoest, N. E. C.: Assessment of model uncertainty for soil moisture through ensemble verification, J. Geophys. Res.-Atmos., 111, 118, doi:10.1029/2005JD006367, 2006.

Di Baldassarre, G. and Montanari, A.: Uncertainty in river discharge observations: a quantitative analysis, Hydrol. Earth Syst. Sci., 13, 913-921, doi:10.5194/hess-13-913-2009, 2009.

Douglas, D. H. and Peucker, T. K.: Algorithms for the Reduction of the Number of Points Required To Represent a Digitized Line or Its Caricature, Cartogr. Int. J. Geogr. Inf. Geovisual., 10, 112 122, doi:10.3138/FM57-6770-U75U-7727, 1973.

Drabek, U.: Anwendungsbezogene Aspekte der operationellen Durchflussvorhersage, $\mathrm{PhD}$ thesis, Institut für Wasserbau und Ingenieurhydrologie, Technische Universität Wien, Wien, 2010.

Duan, Q., Ajami, N. K., Gao, X., and Sorooshian, S.: Multi-model ensemble hydrologic prediction using Bayesian model averaging, Adv. Water Resour., 30, 1371-1386, 2007.

Eckhardt, K.: How to construct recursive digital filters for baseflow separation, Hydrol. Process., 19, 507-515, doi:10.1002/hyp.5675, 2005.

Efstratiadis, A. and Koutsoyiannis, D.: One decade of multi-objective calibration approaches in hydrological modelling: a review, Hydrolog. Sci. J., 55, 58-78, doi:10.1080/02626660903526292, 2010. 
Ehret, U.: Structogram: A method to describe structuredness and complexity of data sets, Math. Geosci., submitted, 2016.

Ehret, U. and Seibert, S. P.: The Series Distance matlab code, GitHub repository, doi:10.5281/zenodo.60356, avalable at: https: //github.com/KIT-HYD/SeriesDistance, 2016.

Ehret, U. and Zehe, E.: Series distance - An intuitive metric to quantify hydrograph similarity in terms of occurrence, amplitude and timing of hydrological events, Hydrol. Earth Syst. Sci., 15, 877896, doi:10.5194/hess-15-877-2011, 2011.

Euser, T., Winsemius, H. C., Hrachowitz, M., Fenicia, F., Uhlenbrook, S., and Savenije, H. H. G.: A framework to assess the realism of model structures using hydrological signatures, Hydrol. Earth Syst. Sci., 17, 1893-1912, doi:10.5194/hess-17-18932013, 2013.

Ewen, J.: Hydrograph Matching Method for Measuring Model Performance, J. Hydrol., 408, 178-187, 2011.

Franz, K. J. and Hogue, T. S.: Evaluating uncertainty estimates in hydrologic models: Borrowing measures from the forecast verification community, Hydrol. Earth Syst. Sci., 15, 3367-3382, doi:10.5194/hess-15-3367-2011, 2011.

Gassmann, M., Stamm, C., Olsson, O., Lange, J., Kümmerer, K., and Weiler, M.: Model-based estimation of pesticides and transformation products and their export pathways in a headwater catchment, Hydrol. Earth Syst. Sci., 17, 5213-5228, doi:10.5194/hess-17-5213-2013, 2013.

Georgakakos, K. P., Seo, D. J., Gupta, H., Schaake, J., and Butts, M. B.: Towards the characterization of streamflow simulation uncertainty through multimodel ensembles, J. Hydrol., 298, 222241, doi:10.1016/j.jhydrol.2004.03.037, 2004.

Gneiting, T., Stanberry, L. I., Grimit, E. P., Held, L., and Johnson, N. A.: Assessing probabilistic forecasts of multivariate quantitites, with an application to ensemble pprediction of surface winds, Test, 17, 211-235, 2008.

Gupta, H. V., Sorooshian, S., and Yapo, P. O.: Toward improved calibration of hydrologic models: Multiple and noncommensurable measures of information, Water Resour. Res., 34, 751-763, doi:10.1029/97WR03495, 1998.

Gupta, H. V., Kling, H., Yilmaz, K. K., and Martinez, G. F.: Decomposition of the mean squared error and NSE performance criteria: Implications for improving hydrological modelling, J. Hydrol., 377, 80-91, doi:10.1016/j.jhydrol.2009.08.003, 2009.

Haag, I., Vollmer, S., and Heß, S.: Aufstellung eines Wasserhaushaltsmodells für das Einzugsgebiet der Iller, Erläuterungsbericht, Auftraggeber Wasserwirtschaftsamt Kempten, Tech. rep., unpublished report, 2005.

Harmel, R. D., Smith, P. K., Migliaccio, K. W., Chaubey, I., Douglas-Mankin, K. R., Benham, B., Shukla, S., MuñozCarpena, R., and Robson, B. J.: Evaluating, interpreting, and communicating performance of hydrologic/water quality models considering intended use: A review and recommendations, Environ. Model. Softw., 57, 40-51, doi:10.1016/j.envsoft.2014.02.013, 2014.

Hrachowitz, M., Fovet, O., Ruiz, L., Euser, T., Gharari, S., Nijzink, R., Freer, J., Savenije, H. H. G., and Gascuel-Odoux, C.: Process consistency in models: The importance of system signatures, expert knowledge, and process complexity, Water Resour. Res., 50, 7445-7469, doi:10.1002/2014WR015484, 2014.

Jacquin, A. P. and Shamseldin, A. Y.: Development of a possibilistic method for the evaluation of predictive uncertainty in rainfall-runoff modeling, Water Resour. Res., 43, W04425, doi:10.1029/2006WR005072, 2007.

Kelleher, C., Wagener, T., and McGlynn, B.: Model-based analysis of the influence of catchment properties on hydrologic partitioning across five mountain headwater sub-catchments, Water Resour. Res., 51, 2-31, doi:10.1002/acr.22212, 2015.

Kollat, J. B., Reed, P. M., and Wagener, T.: When are multiobjective calibration trade-offs in hydrologic models meaningful?, Water Resour. Res., 48, W03520, doi:10.1029/2011WR011534, 2012.

Krzysztofowicz, R.: Bayesian forecasting via deterministic model, Risk Anal., 19, 739-749, doi:10.1023/A:1007050023440, 1999.

Krzysztofowicz, R. and Kelly, K. S.: Hydrologic uncertainty processor for probabilistic river stage forecasting, Water Resour. Res., 36, 3265, doi:10.1029/2000WR900108, 2000.

Laio, F. and Tamea, S.: Verification tools for probabilistic forecasts of continuous hydrological variables, Hydrol. Earth Syst. Sci., 11, 1267-1277, doi:10.5194/hess-11-1267-2007, 2007.

Landesanstalt für Umwelt, Messung und Naturschutz BadenWürttemberg (LUBW): Wasserhaushaltsmodell LARSIM, Hochwasservorhersagezentrale Baden-Württemberg, Karlsruhe, Germany, available at: www.larsim.info/, last access: August 2016.

Legates, D. R. and McCabe, G. J.: Evaluating the use of 'goodness-of-fit' measures in hydrologic and hydroclimatic model validation, Water Resour. Res., 35, 233-241, doi:10.1029/1998WR900018, 1999.

Liu, Y., Brown, J., Demargne, J., and Seo, D. J.: A wavelet-based approach to assessing timing errors in hydrologic predictions, J. Hydrol., 397, 210-224, doi:10.1016/j.jhydrol.2010.11.040, 2011.

Ludwig, K. and Bremicker, M.: The Water Balance Model LARSIM, Tech. Rep. 22, Institut für Hydrologie der Universität Freiburg i. Br., Freiburg i. Br., 2006.

Merz, R. and Blöschl, G.: A regional analysis of event runoff coefficients with respect to climate and catchment characteristics in Austria, Water Resour. Res., 45, 1-19, doi:10.1029/2008WR007163, 2009.

Montanari, A.: What do we mean by 'uncertainty'? The need for a consistent wording about uncertainty assessment in hydrology, Hydrol. Process., 21, 841-845, doi:10.1002/hyp.6623, 2007.

Montanari, A. and Grossi, G.: Estimating the uncertainty of hydrological forecasts: A statistical approach, Water Resour. Res., 44, 1-9, doi:10.1029/2008WR006897, 2008.

Montanari, A., Rosso, R., and Taqqu, M. S.: Fractionally differenced ARIMA models applied to hydrologic time series: Identification, estimation, and simulation, Water Resour. Res., 33, 1035 1044, doi:10.1029/97WR00043, 1997.

Moriasi, D., Arnold, J., Van Liew, M., Binger, R., Harmel, R., and Veith, T.: Model evaluation guidelines for systematic quantification of accuracy in watershed simulations, T. ASABE, 50, 885900, doi:10.13031/2013.23153, 2007.

Mosler, K.: Depth Statistics, in: Robustness Complex Data Struct, edited by: Becker, C., Fried, R., and Kuhnt, S., Springer-Verlag, Berlin, Heidelberg, 17-35, doi:10.1007/978-3642-35494-6, 2013.

Nash, J. E. and Sutcliffe, J. V.: River flow forecasting through conceptual models part I - A discussion of principles, J. Hydrol., 10, 282-290, doi:10.1016/0022-1694(70)90255-6, 1970. 
Nasseri, M., Ansari, A., and Zahraie, B.: Uncertainty assessment of hydrological models with fuzzy extension principle: Evaluation of a new arithmetic operator, Water Resour. Res., 50, 1095-1111, doi:10.1002/2012WR013382, 2014.

Neuweiler, I. and King, P.: Coarse graining of the solute concentration probability distribution for advective transport in porous media, in: Proc. 14th Int. Conf. Comput. Methods Water Resour., edited by: Hassanizadeh, S. M., Schotting, R. J., Gray, W. G., and Pinder, G. F., Elsevier Science Publishers B.V., Delft, 11471154, 2002.

Norbiato, D., Borga, M., Merz, R., Blöschl, G., and Carton, A.: Controls on event runoff coefficients in the eastern Italian Alps, J. Hydrol., 375, 312-325, doi:10.1016/j.jhydrol.2009.06.044, 2009.

Pachepsky, Y., Guber, A., Jacques, D., Simunek, J., Van Genuchten, M. T., Nicholson, T., and Cady, R.: Information content and complexity of simulated soil water fluxes, Geoderma, 134, 253-266, doi:10.1016/j.geoderma.2006.03.003, 2006.

Pappenberger, F. and Beven, K. J.: Functional classification and evaluation of hydrographs based on Multicomponent Mapping (Mx), Int. J. River Basin Manage., 2, 89-100, doi:10.1080/15715124.2004.9635224, 2004.

Perng, C.-S., Wang, H., Zhang, S., and Parker, D.: Landmarks: a new model for similarity-based pattern querying in time series databases, Proc. 16th Int. Conf. Data Eng. (Cat. No. 00CB37073), San Diego, CA, 33-42, doi:10.1109/ICDE.2000.839385, 2000.

Reusser, D. E. and Zehe, E.: Inferring model structural deficits by analyzing temporal dynamics of model performance and parameter sensitivity, Water Resour. Res., 47, W07550, doi:10.1029/2010WR009946, 2011.

Reusser, D. E., Blume, T., Schaefli, B., and Zehe, E.: Analysing the temporal dynamics of model performance for hydrological models, Hydrol. Earth Syst. Sci., 13, 999-1018, doi:10.5194/hess-13999-2009, 2009.

Roulston, M. S. and Smith, L. A.: Combining dynamical and statistical ensembles, Tellus A, 55, 16-30, doi:10.1034/j.16000870.2003.201378.x, 2003.

Schaefli, B. and Gupta, H. V.: Do Nash values have value?, Hydrol. Process., 21, 2075-2080, doi:10.1002/hyp.6825, 2007.

Seibert, J.: On the need for benchmarks in hydrological modelling, Hydrol. Process., 15, 1063-1064, doi:10.1002/hyp.446, 2001.

Seibert, S. P. and Ehret, U.: Detection of flood events in hydrological discharge time series (EGU2012-5924), Geophys. Res. Abstr., vol. 14, 2012.

Seibert, S. P., Skublics, D., and Ehret, U.: The potential of coordinated reservoir operation for flood mitigation in large basins - A case study on the Bavarian Danube using coupled hydrological-hydrodynamic models, J. Hydrol., 517, 1128-1144, doi:10.1016/j.jhydrol.2014.06.048, 2014.
Seibert, S. P., Jackisch, C., Ehret, U., Pfister, L., and Zehe, E.: Exploring the interplay between state, structure and runoff behaviour of lower mesoscale catchments, Hydrol. Earth Syst. Sci. Discuss., doi:10.5194/hess-2016-109, in review, 2016.

Shrestha, D. L., Kayastha, N., and Solomatine, D. P.: A novel approach to parameter uncertainty analysis of hydrological models using neural networks, Hydrol. Earth Syst. Sci., 13, 1235-1248, doi:10.5194/hess-13-1235-2009, 2009.

Stedinger, J. R., Vogel, R. M., Lee, S. U., and Batchelder, R.: Appraisal of the generalized likelihood uncertainty estimation (GLUE) method, Water Resour. Res., 44, 1-17, doi:10.1029/2008WR006822, 2008.

Tukey, J.: Mathematics and Picturing Data, in: Proc. 1974 Congr. Math., vol. 2, edited by: James, R., Vancouver, 523-531, 1975.

Vrugt, J. A., Gupta, H. V., Bastidas, L. A., and Bouten, W.: Effective and efficient algorithm for multiobjective optimization of hydrologic models, Water Resour. Res., 39, 1-19, doi:10.1029/2002WR001746, 2003.

Vrugt, J. A., ter Braak, C. J. F., Clark, M. P., Hyman, J. M., and Robinson, B. A.: Treatment of input uncertainty in hydrologic modeling: Doing hydrology backward with Markov chain Monte Carlo simulation, Water Resour. Res., 44, W00B09, doi:10.1029/2007WR006720, 2008.

Wagener, T., McIntyre, N., Lees, M. J., Wheater, H. S., and Gupta, H. V.: Towards reduced uncertainty in conceptual rainfall-runoff modelling: Dynamic identifiability analysis, Hydrol. Process., 17, 455-476, 2003.

Willmott, C. J.: On the validation of models, Phys. Geogr., 2, 184 194, doi:10.1080/02723646.1981.10642213, 1981.

Wrede, S., Fenicia, F., Martínez-Carreras, N., Juilleret, J., Hissler, C., Krein, A., Savenije, H. H. G., Uhlenbrook, S., Kavetski, D., and Pfister, L.: Towards more systematic perceptual model development: a case study using 3 Luxembourgish catchments, Hydrol. Process., 2750, 2731-2750, doi:10.1002/hyp.10393, 2014.

Zappa, M., Fundel, F., and Jaun, S.: A 'Peak-Box' approach for supporting interpretation and verification of operational ensemble peak-flow forecasts, Hydrol. Process., 27, 117-131, doi:10.1002/hyp.9521, 2013.

Zhang, Y. Y., Shao, Q. X., Ye, A. Z., Xing, H. T., and Xia, J.: Integrated water system simulation by considering hydrological and biogeochemical processes: model development, with parameter sensitivity and autocalibration, Hydrol. Earth Syst. Sci., 20, 529 553, doi:10.5194/hess-20-529-2016, 2016. 